Classification

Physics Abstracts

$75.10 \mathrm{H}-05.50-31.30 \mathrm{~N}-76.80$

\title{
Ising-like model for the two-step spin-crossover
}

A. Bousseksou $\left({ }^{1}\right)$, J. Nasser $\left({ }^{1,2}\right)$, J. Linares $\left({ }^{1}\right)$, K. Boukheddaden $\left({ }^{1}\right)$ and F. Varret $\left({ }^{1}\right)$

( $\left.{ }^{1}\right)$ Département de Recherches Physiques, UA CNRS 71, Université Pierre et Marie Curie, F-75252 Paris Cedex 05, France

$\left({ }^{2}\right)$ Service de Physique du Solide et de Résonance Magnétique, CEN Saclay, F-91191 Gif-surYvette Cedex, France

(Received 10 April 1991, revised 28 November 1991 and 17 February 1992, accepted 27 March 1992)

Résumé . - Nous avons analysé un modèle de type Ising, à deux sous-réseaux couplés "antiferromagnétiquement" , dans l'approximation du champ moyen. Ce modèle permet de bien reproduire les transitions de spin "en deux étapes", dont nous donnons une définition précise . Lorsque les deux sous-réseaux sont équivalents, il implique une brisure spontanée de symétrie qui peut intervenir dans un domaine de température limité par deux "températures de Néel". De plus, lorsqu ils sont inéquivalents, il prédit le renversement simultané de l" "aimantation" des deux sous-réseaux pour une valeur "caractéristique" de la température. Nous avons analysé en détail l'ensemble de ces effets. Ce modèle nous a permis d'ajuster et de discuter les résultats expérimentaux disponibles concernant $\left[\mathrm{Fe}(2-\text { pic })_{3}\right] \mathrm{C} \ell_{2}-\mathrm{EtOH}$ et $\mathrm{Fe}^{\mathrm{II}}\left[5 \mathrm{NO}_{2}-\right.$ sal $\left.-\mathrm{N}(1,4,7,10)\right]$.

\begin{abstract}
We have analyzed an Ising-like model, in the mean-field approach, involving two "antiferromagnetically" coupled sublattices. This model simulates the so-called "two-step" spincrossover transition, for which a precise definition is given. If both sublattices are equivalent, it implies a spontaneous breaking of symmetry which may occur within a temperature range limited by two "Néel températures". It, also predicts a simultaneous reversal of the magnetization of the sublattices (if they are unequivalent) at a "characteristic" value of temperature. These features are analyzed simultaneously with some details. The present model fits and explains well the available experimental data concerning $\left[\mathrm{Fe}(2-\text { pic })_{3}\right] \mathrm{C} \ell_{2}-\mathrm{EtOH}$ and $\mathrm{Fe}^{\mathrm{II}}\left[5 \mathrm{NO}_{2}-\mathrm{sal}-\right.$ $\mathrm{N}(1,4,7,10)]$.
\end{abstract}

\section{Introduction.}

Recently an "unusual" low-spin $\rightleftharpoons$ high-spin (LS $\rightleftharpoons \mathrm{HS}$ ) conversion of $\mathrm{Fe}^{\mathrm{II}}$ has been observed in some molecular solids [1-3]. In these compounds, the fraction $n_{\mathrm{HS}}$ of molecules in the HS state increases with temperature in two steps with a plateau of a few Kelvins. In this report we propose a model which simulates such a behaviour. This model is based on the approach 
of discrete levels [4] which offers a fruitful comparison to magnetic models.

It is well known that, for an isolated molecule, the LS state is lower in energy than the HS state if the ligand field is stronger than the mean spin-pairing energy. The LS $\rightleftharpoons$ HS conversion is thermally possible if the energy distance $\delta$ between the HS and the LS states is of the same order as the thermal energy $k T$ [5].

In the discrete level approach (adapted here from [4]), for the isolated molecule, the Hamiltonian is taken as:

$$
\hat{\mathcal{H}}=\frac{1}{2} \delta \sigma,
$$

where $\sigma$ is a fictitious spin which has two eigen-values, $\mp 1$, corresponding to the LS and HS states respectively. The degeneracies $g_{\mathrm{a}}, g_{\mathrm{b}}$ of the LS, HS states are an essential element of the model; $g_{\mathrm{b}}$ is larger than $g_{\mathrm{a}}$ because of electronic and vibrational properties. In this formalism, the spin conversion is characterized by the mean value of $\sigma$ :

$$
\begin{gathered}
\langle\sigma\rangle=-n_{\mathrm{LS}}+n_{\mathrm{HS}} \quad \text { with } \quad n_{\mathrm{LS}}+n_{\mathrm{HS}}=1, \\
\text { so that } n_{\mathrm{HS}}=\frac{1}{2}(1+\langle\sigma\rangle) .
\end{gathered}
$$

at $0 \mathrm{~K},\langle\sigma\rangle=-1$, all the molecules are in the LS state $\left(n_{\mathrm{HS}}=0\right)$;

at $T \rightarrow \infty,\langle\sigma\rangle \rightarrow \frac{-g_{\mathrm{a}}+g_{\mathrm{b}}}{g_{\mathrm{a}}+g_{\mathrm{b}}} \simeq 1$ if $g_{\mathrm{b}}>>g_{\mathrm{a}} ;$ nearly all the molecules are in the HS state $\left(n_{\mathrm{HS}} \simeq 1\right)$.

A characteristic temperature for the spin conversion, $T_{\mathrm{C}}$, is defined by $k T_{\mathrm{C}} \operatorname{Ln}\left(\frac{g_{\mathrm{b}}}{g_{\mathrm{a}}}\right)=\delta$, which corresponds to $\langle\sigma\rangle=0\left(n_{\mathrm{HS}}=0.5\right)$. In the scope of the present analysis, it is pointed out that the steepest (and major) part of the thermal variation of $\langle\sigma\rangle$, occurs around $T_{\mathrm{C}}$.

In the crystal, the interaction $W_{i j}$ between molecules $i$ and $j$ is a function of both spins $\sigma_{i}$ and $\sigma_{j}$. The general development of this function to the second order is:

$$
\mathbf{W}_{i j}=A_{i j}\left(\sigma_{i}+\sigma_{j}\right)+J_{i j} \sigma_{i} \cdot \sigma_{j}
$$

So the total Hamiltonian is:

$$
\mathcal{H}=\sum_{i} \frac{1}{2} \Delta_{i} \sigma_{i}+\sum_{\{i, j\rangle} J_{i j} \sigma_{i} \cdot \sigma_{j}
$$

where $\sum_{i}$ is a sum over sites, and $\sum_{\langle i, j\rangle}$ a sum over pairs of sites.

In the expression of $\mathcal{H}$, the values of the parameters $\Delta_{i}$ are directly related to the crystal field on the site $i$. This field is the sum of the ligand field of the molecule $i$ and of the crystal field created by the other molecules of the crystal.

The parameters $\Delta_{i}$ and $J_{i j}$ are considered here as phenomenological, and for simplicity we shall consider $\Delta_{i}=\Delta$ for all sites; then $\mathcal{H}$ is an Ising-like Hamiltonian with field (the difference with the true Ising model is $g_{\mathrm{a}} \neq g_{\mathrm{b}}$ ). The case of inequivalent sites will be described by unequal $J_{i j}$ values. It is worth noticing here that a two-step curve can be trivially obtained by considering two independent sublattices with unequal values of $\Delta_{i}$. The present paper, considering equal $\Delta_{i}$, is then focused on the role of the intermolecular interactions in the double-step transitions. 
Wajnflasz and Pick [4] have studied, in the mean-field approximation, the "ferromagnetic" case of a single sublattice characterized by a single "exchange" parameter $J<0$. They found that the crystal displays a first order transition at the temperature $T_{\mathrm{C}}$, now defined as $k T_{\mathrm{c}} \operatorname{Ln}\left(\frac{g_{\mathrm{b}}}{g_{\mathrm{a}}}\right)=\Delta$, if the interaction parameter $|J|$ is larger than the threshold value $k T_{\mathrm{C}}$; typical plots of $\langle\sigma(T)\rangle$ are reproduced in section 2 , figure 1 . The occurrence of such a transition is clearly due to the degeneracies $g_{\mathrm{b}} \neq g_{\mathrm{a}}$, since the ferromagnetic Ising model under field does not lead to a phase transition (for $g_{\mathrm{b}} \rightarrow g_{\mathrm{a}}, T_{\mathrm{c}} \rightarrow \infty$ ).

On the other hand, it is remarkable that the first-order transition is not accompanied by a symmetry change in the crystal; this is because symmetry is already broken by the "field" $($ parameter $\Delta$ ).

In the present study, we analyse the "antiferromagnetic" and "ferrimagnetic" cases, involving two sublattices, also in the mean-field approach. It is known [6] that the antiferromagnetic Ising model under magnetic field can lead to a para-antiferromagnetic phase transition. In the spinconversion system, the corresponding effect is a spontaneous breaking of symmetry between the sublattices (when they are structurally equivalent), which has been briefly described by Bari and Sivardière [7]. We report here a detailed analysis of the symmetry breaking and of the two-step character of the transition, the latter point being the initial goal of the present study.

The present paper contains the description and analysis of the model (Sect. 2), the comparison with a previous study of Bari and Sivardière [7] (Sect. 3) and the application of the present model to the available experimental data (Sect. 4). The latter section is also devoted to the conclusion.

For convenience, we shall refer to Wajnsflasz and Pick, Bari and Sivardière, as W-P, B-S respectively. The notation is used are presented on a recapitulary table (Tab. I), which also summarizes the results of the present analysis

Table I. - List of notations and summary of the results of the present analysis.

\section{Notation}

$r=\frac{g_{\mathrm{a}}}{g_{\mathrm{b}}}$

$T_{\mathrm{C}}$ (Eq. (4)): simultaneous sign change of $\left\langle\sigma_{\mathrm{A}}\right\rangle,\left\langle\sigma_{\mathrm{B}}\right\rangle$ (if $J_{\mathrm{A}} \neq J_{\mathrm{B}}$ ) and sign change of $\langle\sigma\rangle$

$T_{\mathrm{CA}}^{-}, T_{\mathrm{CA}}^{+}$: sign changes of $\left\langle\sigma_{\mathrm{A}}\right\rangle$

$T_{\mathrm{CB}}^{-}, T_{\mathrm{CB}}^{+}$: sign changes of $\left\langle\sigma_{\mathrm{B}}\right\rangle$

$$
\text { if }\left|J_{A}\right|>\left|J_{B}\right|
$$

$T_{\mathrm{CB}}^{-}, T_{\mathrm{CB}}^{+}$exist if $J_{\mathrm{AB}}>\left(J_{\mathrm{AB}}\right)_{\mathrm{s}}$

$T_{\mathrm{CB}}^{-}<T_{\mathrm{CA}}^{-}<T_{\mathrm{CA}}^{+}<T_{\mathrm{CB}}^{+}$

$T_{\mathrm{CA}}^{-}, T_{\mathrm{CA}}^{+}$have no physical relevance

$T_{\mathrm{CB}}^{-} \neq T_{\mathrm{CB}}^{+}$define the two-step character

two possibilities:

(i) $T_{\mathrm{CB}}^{-}<T_{\mathrm{C}}<T_{\mathrm{CB}}^{+}$

(ii) $T_{\mathrm{CB}}^{-}<T_{\mathrm{CB}}^{+}<T_{\mathrm{C}}$

the continuous / discontinuous character of conversions changes:

for $T_{\mathrm{CB}}^{-}$at $\left(J_{\mathrm{AB}}\right)_{\mathrm{d}_{1}}$ if $\left|J_{\mathrm{B}}\right|<k T_{\mathrm{C}}$

for $T_{\mathrm{CB}}^{+}$at $\left(J_{\mathrm{AB}}\right)_{\mathrm{d}_{2}}$ if $\left|J_{\mathrm{B}}\right|>k T_{\mathrm{C}}$ 


$$
J_{\mathbf{A}}=J_{\mathbf{B}}
$$

$T_{\mathrm{CA}}^{-}=T_{\mathrm{CB}}^{-}=T_{\mathrm{C}}^{-} ; \quad T_{\mathrm{CA}}^{+}=T_{\mathrm{CB}}^{+}=T_{\mathrm{C}}^{+}$

$T_{\mathrm{N}}^{-}, T_{\mathrm{N}}^{+}$: limiting temperatures of possible asymmetrical solutions.

In principle:

$T_{\mathrm{N}}^{-} \leq T_{\mathrm{C}}^{-}<T_{\mathrm{C}}^{+} \leq T_{\mathrm{N}}^{+}$

or $T_{\mathrm{N}}^{-}<T_{\mathrm{N}}^{+} \leq T_{\mathrm{C}}$ if $T_{\mathrm{C}}^{-}, T_{\mathrm{C}}^{+} \neq T_{\mathrm{C}}$ do not exist.

\section{Analysis of the model.}

We start with the Hamiltonian:

$$
\hat{\mathcal{K}}=\sum_{i} \frac{1}{2} \Delta \sigma_{i}+\sum_{\langle i, j\rangle} J_{i j} \sigma_{i} \cdot \sigma_{j}
$$

where the fictitious spins $\sigma$ have eigenvalues $\mp 1$, associated with degeneracies $g_{\mathrm{a}} \neq g_{\mathrm{b}}$.

We consider two sublattices A,B containing the same number of molecules, such that:

$$
\begin{gathered}
n_{\mathrm{HS}}^{(\mathrm{A}, \mathrm{B})}=\frac{1}{2}\left(1+\left\langle\sigma_{\mathrm{A}, \mathrm{B}}\right\rangle\right) \\
n_{\mathrm{HS}}=\frac{1}{2}\left(n_{\mathrm{HS}}^{(\mathrm{A})}+n_{\mathrm{HS}}^{(\mathrm{B})}\right)=\frac{1}{4}(2+\langle\sigma\rangle), \\
\text { where }\langle\sigma\rangle=\left\langle\sigma_{\mathrm{A}}\right\rangle+\left(\sigma_{\mathrm{B}}\right\rangle .
\end{gathered}
$$

Let $J_{\mathrm{A}}, J_{\mathrm{B}}, J_{\mathrm{AB}}$ be the intra- and inter-sublattice interaction parameters (including the number of neighbours). In the present study we found that the onset of a double-step transition required intra- and inter-sublattice interactions, respectively "ferromagnetic" and "antiferromagnetic":

$$
J_{\mathrm{A}}, J_{\mathrm{B}}<0, J_{\mathrm{AB}}>0 \text {. }
$$

In the mean-field approach $\hat{\mathcal{K}}$ is replaced by one-site Hamiltonians:

$$
\begin{aligned}
& \hat{\mathcal{H}}_{\mathrm{A}}=\frac{\Delta}{2} \cdot \sigma_{\mathrm{A}}+\sigma_{\mathrm{A}} \cdot\left(J_{\mathrm{A}}\left\langle\sigma_{\mathrm{A}}\right\rangle+J_{\mathrm{AB}}\left\langle\sigma_{\mathrm{B}}\right\rangle\right) \\
& \hat{\mathcal{H}}_{\mathrm{B}}=\frac{\Delta}{2} \cdot \sigma_{\mathrm{B}}+\sigma_{\mathrm{B}} \cdot\left(J_{\mathrm{B}}\left\langle\sigma_{\mathrm{B}}\right\rangle+J_{\mathrm{AB}}\left\langle\sigma_{\mathrm{A}}\right\rangle\right)
\end{aligned}
$$

Equations (2a,2b) are formally similar to the mean-field equation derived by Wajnflasz and Pick [4] in the one-sublattice model: the crystal-field value $\Delta$ is merely shifted by the constant value $2 J_{\mathrm{AB}}\left\langle\sigma_{\mathrm{B}, \mathrm{A}}\right\rangle$, respectively. The mean-field equations, derived from (2a,2b) are:

$$
\left\langle\sigma_{\mathrm{A}}\right\rangle=\frac{-1+r \exp -\beta\left(\Delta+2 J_{\mathrm{A}}\left\langle\sigma_{\mathrm{A}}\right\rangle+2 J_{\mathrm{AB}}\left\langle\sigma_{\mathrm{B}}\right)\right)}{1+r \exp -\beta\left(\Delta+2 J_{\mathrm{A}}\left\langle\sigma_{\mathrm{A}}\right\rangle+2 J_{\mathrm{AB}}\left\langle\sigma_{\mathrm{B}}\right\rangle\right)}
$$

and

$$
\left\langle\sigma_{\mathrm{B}}\right\rangle=\frac{-1+r \exp -\beta\left(\Delta+2 J_{\mathrm{B}}\left(\sigma_{\mathrm{B}}\right\rangle+2 J_{\mathrm{AB}}\left\langle\sigma_{\mathrm{A}}\right)\right)}{1+r \exp -\beta\left(\Delta+2 J_{\mathrm{B}}\left(\sigma_{\mathrm{B}}\right)+2 J_{\mathrm{AB}}\left\langle\sigma_{\mathrm{A}}\right\rangle\right)}
$$

where $r$ is the ratio $g_{\mathrm{b}} / g_{\mathrm{a}}$, and $\beta=(k T)^{-1}$.

The expression of $F$, the free energy, can be found in the Appendix. 
The particular case $J_{\mathrm{A}}=J_{\mathbf{B}}$ (structurally equivalent sublattices) involves an additional degeneracy associated to the interchange of the sublattices when the symmetry of the system is spontaneously broken; it will be considered after the general case (in Sect. 2.5).

\section{We choose sublattices $A, B$ so that $\left|J_{A}\right|>\left|J_{B}\right|$.}

The thermal evolution of $\left\langle\sigma_{\mathrm{A}}\right\rangle,\left\langle\sigma_{\mathrm{B}}\right\rangle$ is really complex, but can be rationalized by considering "characteristic temperatures" $T_{\mathrm{CA}}, T_{\mathrm{CB}}$ for which the mean spin values change sign in one of the sublattices. In the following, it is shown that each sublattice possesses two values of the so-defined characteristic temperature. In addition, both sublattices change sign simultaneously at $T_{\mathrm{C}}$, defined (as in the single lattice model) by

$$
k T_{\mathrm{C}} \operatorname{Ln}(r)=\Delta
$$

so that the "characteristic temperatures" involved in the present analysis are: $T_{\mathrm{CA}}^{-}, T_{\mathrm{CA}}^{+}, T_{\mathrm{CB}}^{-}$, $T_{\mathrm{CB}}^{+}, T_{\mathrm{C}}$. It is essentially around these temperatures that $\left\langle\sigma_{\mathrm{A}}\right\rangle,\left\langle\sigma_{\mathrm{B}}\right\rangle$ have their steepest (and largest) variations.

We shall firstly determine and classify these temperatures (Sect. 2.1); secondly, we shall consider the behavior at $T_{C}$ (Sect. 2.2). This allows us to sketch the various possibilities for the thermal evolution of the system in the general case (Sect. 2.3). Then the problems of the continuous / discontinuous character of the conversions (Sect. 2.4) and of the spontaneous breaking of symmetry in the case $J_{\mathrm{A}}=J_{\mathrm{B}}$ (Sect. 2.5) will be considered. The results will be collected in section 2.6 in order to provide a general description of the properties of the model.

2.1 Determination OF THE CHARACTERISTIC TEMPERATURES $T_{\mathrm{CA}}, T_{\mathrm{CB}}$. - At first, we recall how the sign change occurs in the single sublattice model (ferromagnetic case) [4]. This is sketched in figure 1: the change occurs at $T_{\mathrm{C}}$, previously defined, and easily characterized by the existence of the trivial solution $\langle\sigma\rangle=0$. For $|J|\left\langle k T_{\mathrm{C}}\right.$, the change occurs continuously and $\langle\sigma\rangle=0$ is effectively reached. For $|J|\rangle k T_{\mathrm{C}}$, there also exist two non-trivial solutions the free-energy values of which are equal at $T_{C}$. In its thermodynamically stable state, the system jumps at $T_{\mathrm{C}}$ from one to the other. The presence of hysteresis, of course, would shift the temperature values.

The same approach, discarding hysteresis effects, in the two sublattice system leads to define the characteristic temperatures $T_{\mathrm{CA}}$ (resp. $T_{\mathrm{CB}}$ ) as the temperature values at which a "trivial" solution with $\left(\sigma_{\mathrm{A}}\right\rangle\left(\mathrm{resp} .\left\langle\sigma_{\mathrm{B}}\right\rangle\right)=0$ exists.

Considering $\left\{\sigma_{B}\right\rangle$ as a function of $T,\left\langle\sigma_{A}\right\rangle$, we deduce from equations $(3 \mathrm{a}, 3 \mathrm{~b})$ that $\left\langle\sigma_{\mathrm{B}}\right\rangle=0$ when the following equations are verified:

$$
\begin{gathered}
k T \operatorname{Ln}(r)=\Delta+2 J_{\mathrm{AB}}\left\langle\sigma_{\mathrm{A}}\right\rangle \\
\left\langle\sigma_{\mathrm{A}}\right\rangle=\frac{-1+r \exp -\beta\left(\Delta+2 J_{\mathrm{A}}\left\langle\sigma_{A}\right\rangle\right)}{1+r \exp -\beta\left(\Delta+2 J_{\mathrm{A}}\left\langle\sigma_{\mathrm{A}}\right\rangle\right)}
\end{gathered}
$$

In the axes $\left\{\left\langle\sigma_{\mathrm{A}}\right\rangle, T\right\}$ equation (5) is represented by a straight line which intersects the $T$ axis at the value $T_{\mathrm{C}}$, and equation (6a) by a curve similar to those of figure 1 . In figure 2 , the graphic resolution of the system $(5,6 \mathrm{a})$ is shown. It has up to three solutions, $T_{\mathrm{C}}, T_{\mathrm{CB}}^{-}, T_{\mathrm{CB}}^{+}$.

$T_{\mathrm{CB}}^{ \pm}$are the non-trivial (i.e. $\neq T_{\mathrm{C}}$ ) solutions of the system $(5,6 \mathrm{a})$. They exist when $J_{\mathrm{AB}}$ is larger than a threshold value $\left(J_{\mathrm{AB}}\right)_{\mathrm{s}}$. Due to the negative curvature at $T=T_{\mathrm{C}}$ of the curve representing equation $(6 a)$, it is concluded that:

$$
T_{\mathrm{CB}}^{-}<T_{\mathrm{C}} ; T_{\mathrm{CB}}^{+} \lessgtr T_{\mathrm{C}}
$$




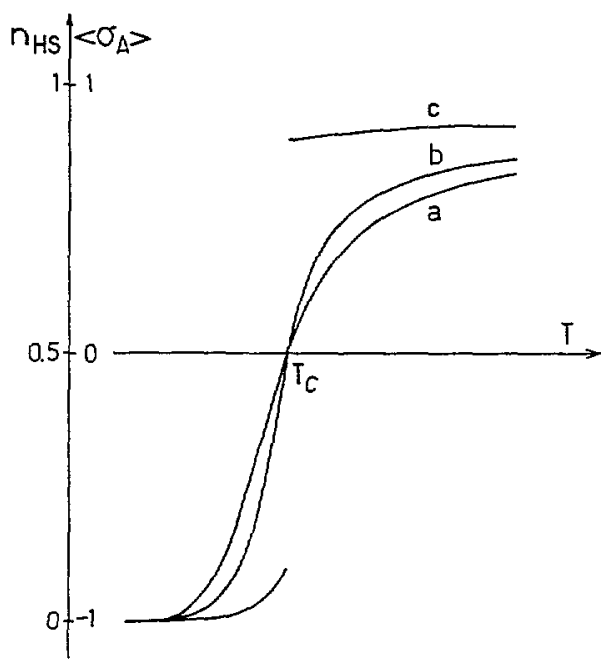

Fig. 1. - Computed curves from equation (6a) for different $J_{\mathrm{A}}$ values, with $r=\frac{g_{\mathrm{b}}}{g_{\mathrm{a}}}=15$; these curves also hold for the single-sublattice model of [4]. $\frac{J_{A}}{k T_{C}}=-0.40(a),-0.67(b),-1.33$ (c)

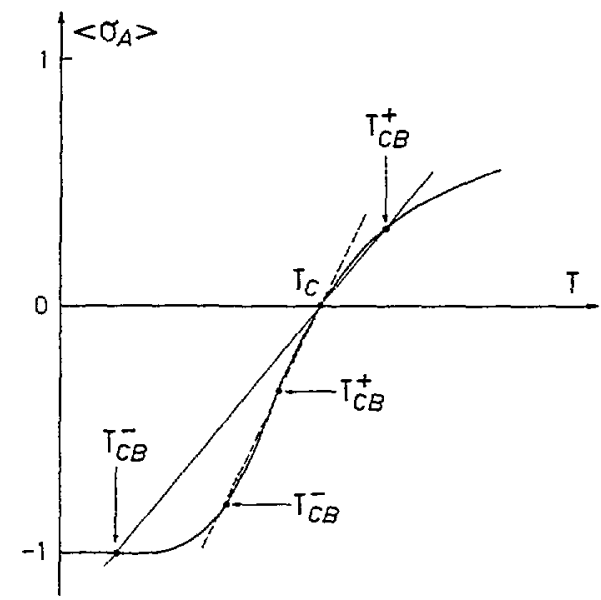

Fig. 2. - Graphic resolution of the system $(5,6 \mathrm{a})$. When the curve is continuous at $T_{\mathrm{C}}$ and when the slope of the straight line is small enough (large $J_{\mathrm{AB}}$ ), there are three solutions: $T_{\mathrm{CB}}^{ \pm}, T_{\mathrm{C}}$, with $T_{\mathrm{CB}}^{-}<T_{\mathrm{C}}, T_{\mathrm{CB}}^{+} \gtrless T_{\mathrm{C}}$.

The "trivial" solution is:

at $T=T_{\mathrm{CB}}^{-} \cdot\left\langle\sigma_{\mathrm{B}}\right\rangle=0,\left\langle\sigma_{\mathrm{A}}\right\rangle=\left\langle\sigma_{\mathrm{A}}\right\rangle_{\left\{\sigma_{B}\right\rangle=0}^{-}<0$,

at $T=T_{\mathrm{CB}}^{+} \cdot\left\langle\sigma_{\mathrm{B}}\right\rangle=0,\left\langle\sigma_{\mathrm{A}}\right\rangle=\left\langle\sigma_{\mathrm{A}}\right\rangle_{\left(\sigma_{\mathrm{B}}\right)=0}^{+} \lessgtr 0$, according to $T_{\mathrm{CB}}^{+} \lessgtr T_{\mathrm{C}}$, respectively.

Because of the self-consistent treatment of the system $(5,6 \mathrm{a})$, the above relations can be considered as sign conditions which have to be satisfied for the (continuous) sign change 
effectively to occur. When the concerned sign condition is not satisfied, the sign of the magnetization does not change, and the corresponding characteristic temperature looses its physical relevance. These sign conditions will be of major importance in section 2.3 .

We consequently distinguish two cases:

(i) $T_{\mathrm{CB}}^{-}<T_{\mathrm{C}}<T_{\mathrm{CB}}^{+}$,

(ii) $T_{\mathrm{CB}}^{-}<T_{\mathrm{CB}}^{+}<T_{c}$.

By considering the slope at $T=T_{C}$ of the curve representing equation (6a), we easily show that case (ii) requires $J_{\mathrm{AB}}<k T_{\mathrm{C}}+J_{\mathrm{A}}$. When the curve given by (Eq. (6a)) is discontinuous at $T_{\mathrm{C}}$ (for $\left|J_{\mathrm{A}}\right|>k T_{\mathrm{C}}$ ), the threshold value $\left(J_{\mathrm{AB}}\right)_{\mathrm{s}}$ is equal to zero. We have plotted in figure 3 the computed values of $\left(J_{\mathrm{AB}}\right)_{\mathrm{s}}$ as a function of $J_{\mathrm{A}}$.

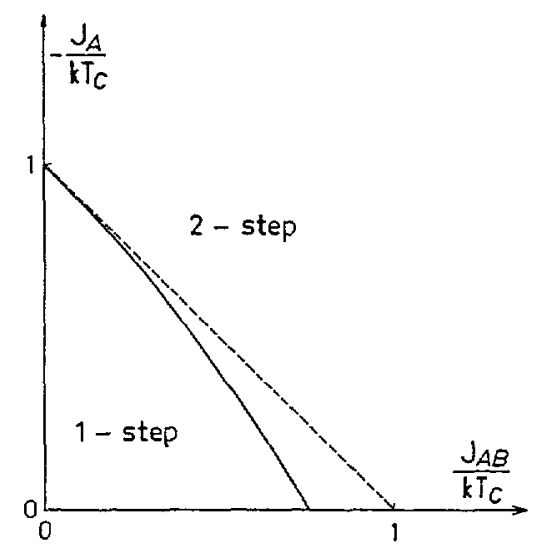

Fig. 3. - Plot of $\left(J_{\mathrm{AB}}\right)_{\mathrm{s}}=f\left(J_{\mathrm{A}}\right)$, in the general case $\left|J_{\mathrm{A}}\right|>\left|J_{\mathrm{B}}\right|$. This is also the phase diagram relative to the one- / two- step character of the conversion. The dashed line separates $T_{\mathrm{CB}}^{+}>T_{\mathrm{C}}$ (above) from $T_{\mathrm{CB}}^{+}<T_{\mathrm{C}}$ (below). This diagram does not depend on $J_{\mathrm{B}}$.

A similar analysis can be performed for the other sublattice, using equation (5) and a modification (6b) of equation (6a) (replacing $A$ by $B$ ). It allows defining two other characteristic temperatures: $T_{\mathrm{CA}}^{-}, T_{\mathrm{CA}}^{+}$. We easily show (see Fig.4) that:

$$
T_{\mathrm{CB}}^{-}<T_{\mathrm{CA}}^{-} \leq T_{\mathrm{CA}}^{+}<T_{\mathrm{CB}}^{+}\left(\text {for }\left|J_{\mathrm{A}}\right|>\left|J_{\mathrm{B}}\right|\right) \text {. }
$$

In fact, for reasons which will appear in the next sections, $T_{C_{A}}^{ \pm}$have no physical relevance, so that the only important temperatures for the sign changes of the magnetization of the sublattices are:

$$
T_{\mathrm{CB}}^{-}<T_{\mathrm{C}} \lessgtr T_{\mathrm{CB}}^{+} \text {. }
$$

We now consider that $T_{\mathrm{CB}}^{-} \neq T_{\mathrm{CB}}^{+}$define the two-step character of the conversion; such a definition applies for any $\left|J_{A}\right| \geq\left|J_{B}\right|$. Then, it is possible to interpret figure 3 as the phase diagram (in $J_{\mathrm{A}}, J_{\mathrm{AB}}$ axes) of the one- / two- step character of the transition. It is worth noting that this diagram does not depend on $J_{B}$ (provided that $\left|J_{A}\right|>\left|J_{B}\right|$, as assumed from the beginning). 


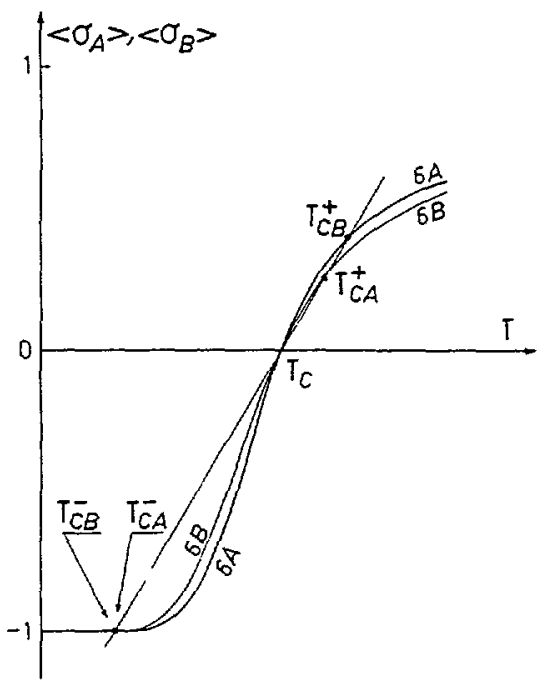

Fig. 4. - Simultaneous graphic determination of $T_{\mathrm{CB}}^{ \pm}$and $T_{\mathrm{CA}}^{ \pm}$, involving two different curves $\left(\left\langle\sigma_{\mathrm{A}}\right\rangle\right.$, $\left\langle\sigma_{\mathrm{B}}\right\rangle$ from respectively equations $(6 \mathrm{a}, 6 \mathrm{~b})$ ), and the same straight line (eq.(5)), showing: $T_{\mathrm{CB}}^{-}<T_{\mathrm{CA}}^{-}<$ $T_{\mathrm{CA}}^{+}<T_{\mathrm{CB}}^{+}\left(\right.$for $\left.\left|J_{\mathrm{A}}\right|>\left|J_{\mathrm{B}}\right|\right)$.

2.2 STUDY AT $T=T_{\mathrm{C}}$. - Applying equation (4) into equations (3a,3b), we obtain

$$
\begin{aligned}
& \left\langle\sigma_{\mathrm{A}}\right\rangle=-\tanh \beta_{\mathrm{C}}\left(J_{\mathrm{A}}\left\langle\sigma_{\mathrm{A}}\right\rangle+J_{\mathrm{AB}}\left\langle\sigma_{\mathrm{B}}\right\rangle\right) \\
& \left\langle\sigma_{\mathrm{B}}\right\rangle=-\tanh \beta_{\mathrm{C}}\left(J_{\mathrm{B}}\left\langle\sigma_{\mathrm{B}}\right\rangle+J_{\mathrm{AB}}\left\langle\sigma_{\mathrm{A}}\right\rangle\right)
\end{aligned}
$$

with $\beta_{\mathrm{C}}=\left(k T_{\mathrm{C}}\right)^{-1}$.

The study of the system $(7 \mathrm{a}, 7 \mathrm{~b})$ leads to a trivial solution and eventually (for $J_{\mathrm{AB}}$ sufficiently large) to a symmetrical pair of non-trivial solutions:

$$
\left(\left\langle\sigma_{\mathrm{A}}\right\rangle=\left\langle\sigma_{\mathrm{B}}\right\rangle=0\right) ;\left(\left\langle\sigma_{\mathrm{A}}\right\rangle \neq 0,\left\langle\sigma_{\mathrm{B}}\right\rangle \neq 0\right) \text { and }\left(-\left\langle\sigma_{\mathrm{A}}\right\rangle,-\left\langle\sigma_{\mathrm{B}}\right\rangle\right) \text {. }
$$

The non-trivial solutions, when they exist, have the same free energy (see Appendix) which is lower than that of the trivial solution (this was controlled by numerical computations). Their entropies are however different, and consequently the values of the free energy of these two solutions cross each other at $T_{\mathrm{C}}$. So, the solution stable below $T_{\mathrm{C}}$ becomes unstable above, and vice-versa: $\left\langle\sigma_{A}\right\rangle,\left\langle\sigma_{B}\right\rangle$, and $\langle\sigma\rangle=\left\langle\sigma_{A}\right\rangle+\left\langle\sigma_{B}\right\rangle$, abruptly change from finite values to their opposites; the crystal displays at $T_{\mathrm{C}}$ a first order phase transition which involves an entropy discontinuity. This does not exactly correspond to an interchange of the sublattices $\left(<\sigma_{\mathrm{A}}\left(T_{\mathrm{C}}\right)>\neq-<\sigma_{\mathrm{B}}\left(T_{\mathrm{C}}\right)>\right.$ ), but to a simultaneous reversal of their magnetizations.

It is worth noting for the following that these discontinuities are related to the unequivalence of the sublattices, and vanish when $J_{\mathrm{A}} \rightarrow J_{\mathrm{B}}$. Then, there is no more thermodynamical reason for a reversal of the magnetizations.

When the trivial solution exists alone, both sublattices change sign continuously at $T_{\mathrm{C}}$.

2.3 THERMal VARIATION OF $\left\langle\sigma_{\mathrm{A}}\right\rangle,\left\langle\sigma_{\mathrm{B}}\right\rangle$ IN THE GENERAL CASE. - The thermal variation is monitored by the numerous sign changes which may occur at $T_{\mathrm{C}}, T_{\mathrm{CA}}^{ \pm}, T_{\mathrm{CB}}^{ \pm}$. It is crucial to 
keep in mind that these sign changes only occur for a given sign of the magnetization of the other sublattice; we recall the sign conditions (Sect. 2.1) for a continuous variation through 0 : at $T=T_{\mathrm{CB}}^{-} \cdot\left\langle\sigma_{\mathrm{A}}\right\rangle<0$; at $\mathrm{T}=T_{\mathrm{CB}}^{+} \lessgtr T_{\mathrm{C}}:\left\langle\sigma_{\mathrm{A}}\right\rangle \lessgtr 0$, and similar relations obtained by interchanging $\mathrm{A}, \mathrm{B}$.

Three possible situations are considered in the case when $T_{\mathrm{CA}}^{ \pm}, T_{\mathrm{CB}}^{ \pm}$exist:

(a) $T_{\mathrm{CB}}^{-}<T_{\mathrm{CA}}^{-}<T_{\mathrm{C}}<T_{\mathrm{CA}}^{+}<T_{\mathrm{CB}}^{+}$;

(b) $T_{\mathrm{CB}}^{-}<T_{\mathrm{CA}}^{-}<T_{\mathrm{CA}}^{+}<T_{\mathrm{C}}<T_{\mathrm{CB}}^{+}$;

(c) $T_{\mathrm{CB}}^{-}<T_{\mathrm{CA}}^{-}<T_{\mathrm{CA}}^{+}<T_{\mathrm{CB}}^{+}<T_{\mathrm{C}}$.

The corresponding changes of signs are easily determined and are displayed in figure 5 (respectively in $a, b, c)$. Here we only report the reasoning for case $(a)$ :

At $0 \mathrm{~K},\left\langle\sigma_{\mathrm{A}}\right\rangle=\left\langle\sigma_{\mathrm{B}}\right\rangle=-1$ : all molecules are low-spin. When temperature is increased, $\left\langle\sigma_{\mathrm{B}}\right\rangle$ changes sign at $T_{\mathrm{CB}}^{-}$where the sign condition $\left\langle\sigma_{\mathrm{A}}\right\rangle<0$ is satisfied: most of molecules $\mathrm{B}$ become high-spin, while most of $\mathrm{A}$ remain low-spin. Up to $T_{\mathrm{C}},\left\langle\sigma_{\mathrm{A}}\right\rangle$ remains negative (most of molecules A remain low-spin); this is because the sign condition at $T_{\mathrm{CA}}^{-}\left(\left\langle\sigma_{\mathrm{B}}\right\rangle<0\right)$ is not satisfied; on the contrary, this condition tells that the temperature at which $\left\langle\sigma_{\mathrm{A}}\right\rangle$ is allowed to change sign is now (and temporarily!) $T_{\mathrm{CA}}^{+}>T_{\mathrm{C}}$. It is easily figured out that the shift in the characteristic value of sublattice $A$ (from $T_{\mathrm{CA}^{-}}^{-}$to $T_{\mathrm{CA}}^{+}$) slows down the thermal variation of sublattice A; in other words, the energy gap of the A-molecules, due to the "field" $\frac{\Delta}{2}+J_{\mathrm{A}}\left\langle\sigma_{\mathrm{A}}\right\rangle+J_{\mathrm{AB}}\left\langle\sigma_{\mathrm{B}}\right\rangle$, is increased by the sign change of the magnetization of sublattice $\mathrm{B}$. This can be considered as the basic mechanism responsible for the occurrence of the plateau.

At $T_{\mathrm{C}}$, as reported in the previous section, both sub-lattices simultaneously change sign.

Above $T_{\mathrm{C}},\left\langle\sigma_{\mathrm{A}}\right\rangle$ remains positive, because the sign condition at $T_{\mathrm{CA}}^{+},\left\langle\sigma_{\mathrm{B}}\right\rangle>0$, is not satisfied. On the contrary, $\left\langle\sigma_{\mathrm{B}}\right\rangle$ which is firstly negative will change sign for a second time, because the sign condition at $T_{\mathrm{CB}}^{+},\left\langle\sigma_{\mathrm{A}}\right\rangle>0$, is satisfied again.

The previous analysis is no longer valid for a discontinuous sign change of $\left(\sigma_{\mathrm{B}}\right)$; however all numerical resolutions performed in the present study have shown thermal behaviors in agreement with the schemes of figure 5 , which indeed reflect all possible two-step behaviors.

The case where the $T_{\mathrm{CA}}^{ \pm}$do not exist leads to behaviors identical to those described above, since nothing happens at these temperatures when they exist. At last, the trivial case where the $T_{\mathrm{CA}, \mathrm{CB}}^{ \pm}$do not exist merely leads to the simultaneous reversals at $T_{\mathrm{C}}$.

The particular case $J_{\mathrm{A}}=J_{\mathrm{B}}$, for which there is no longer a reversal at $T_{\mathrm{C}}$ is sketched in figure $5 \mathrm{~d}$.

For convenience, we consider that $T_{\mathrm{CB}}^{-}, T_{\mathrm{CB}}^{+}$define the temperature range of the plateau. These temperatures are obtained according to equations $(5,6 \mathrm{a})$ :

$$
\begin{aligned}
& T_{\mathrm{CB}}^{-}=2 \frac{J_{\mathrm{AB}}}{k \operatorname{Ln}(r)}\left\langle\sigma_{\mathrm{A}}\right\rangle_{\left(\sigma_{\mathrm{B}}\right\}=0}^{-} \\
& T_{\mathrm{CB}}^{+}=2 \frac{J_{\mathrm{AB}}}{k \operatorname{Ln}(r)}\left\langle\sigma_{\mathrm{A}}\right\rangle_{\left(\sigma_{\mathrm{B}}\right)=0}^{+}
\end{aligned}
$$

We then determine the width of the plateau:

$$
T_{\mathrm{CB}}^{+}-T_{\mathrm{CB}}^{-}=2 \frac{J_{\mathrm{AB}}}{k \operatorname{Ln}(r)}\left(\left\langle\sigma_{\mathrm{A}}\right)^{+}-\left\langle\sigma_{\mathrm{A}}\right\rangle^{-}\right)_{\left\{\sigma_{\mathrm{B}}\right\}=0} \leq 4 \frac{J_{\mathrm{AB}}}{k \operatorname{Ln}(r)}
$$


(a)

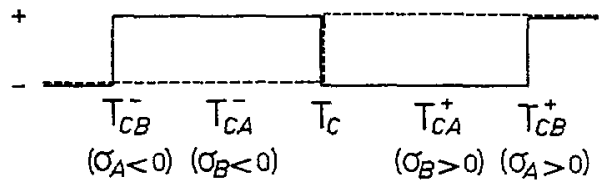

(b)

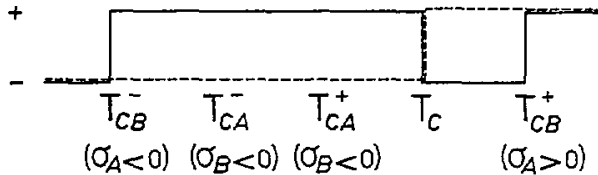

(c)

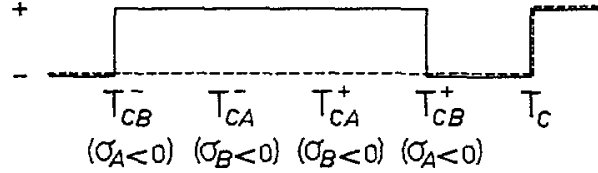

(d)
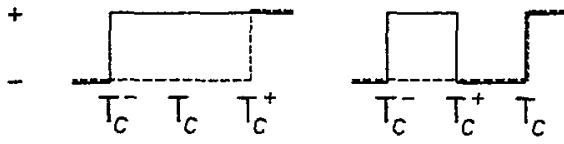

Fig. 5. - Two-step thermal evolution of the signs of $\left\langle\sigma_{A}\right\rangle$ (dashed lines), $\left\langle\sigma_{B}\right\rangle$ (solid lines). It is supposed $\left|J_{A}\right|>\left|J_{B}\right|$. The concerned sign conditions (required for the sign change to occur) have been indicated in brackets. (a), (b), (c) refer to the possible three situations listed in the text (Sect. 2.3). (d) depicts the case $J_{\mathrm{A}}=J_{\mathrm{B}}$; the thermodynamically equivalent solution for which $\mathrm{A}$, $B$ are interchanged has not been drawn. (This diagram holds for both continuous and discontinuous variations of $\left\langle\sigma_{A}, \sigma_{B}\right\rangle$, and does not describe how large is the variation when it is discontinuous).

2.4 CONTINUOUS / DISCONTINUOUS CHARACTER OF THE CONVERSIONS. - To clarify the continuous or discontinuous character of the sign change of $\left\langle\sigma_{\mathrm{B}}\right\rangle$ at $T_{\mathrm{CB}}^{-}$and $T_{\mathrm{CB}}^{+}$, we have followed the analysis developed by Wajnflasz and Pick [4] in the case of a single sublattice: at $T_{\mathrm{CB}}^{ \pm},\left\langle\sigma_{\mathrm{B}}\right\rangle$ varies discontinuously if $k T_{\mathrm{CB}}^{ \pm}<\left|J_{\mathrm{B}}\right|$. Otherwise, it varies continuously and goes through zero at $T_{\mathrm{CB}}^{-}$.

$T_{\mathrm{CB}}^{ \pm}$are functions of $k T_{\mathrm{C}}, J_{\mathrm{AB}}, J_{\mathrm{A}}$; their determination requires the numerical resolution of the system $(5,6 \mathrm{a})$. We have plotted the computed values of $T_{\mathrm{CB}}^{ \pm}$as a function of $J_{\mathrm{AB}}$ for several values of $J_{\mathrm{A}}$. Some of these plots are reported in figures $6 \mathrm{a}, \mathrm{b}$.

Moving upwards in the diagrams, the value of $J_{\mathrm{AB}}$ for the change from discontinuous to continuous has been labelled as follows:

$\left(J_{\mathrm{AB}}\right)_{\mathrm{d}_{1}}$, for the case $\left|J_{\mathrm{B}}\right|<k T_{\mathrm{C}}$ (Fig. 6a);

$\left(J_{\mathrm{AB}}\right)_{\mathrm{d}_{2}}$, for $k T_{\mathrm{C}}<\left|J_{\mathrm{B}}\right|$ (Fig. $6 \mathrm{~b}$ ).

(It can be noted that $\left(J_{\mathrm{AB}}\right)_{\mathrm{d}}$ depends on $k T_{\mathrm{C}}, J_{\mathrm{A}}, J_{\mathrm{B}}$, while $\left(J_{\mathrm{AB}}\right)_{\mathrm{s}}$ on $k T_{\mathrm{C}}, J_{\mathrm{A}}$ only).

Also, it appears clearly from the computations that any discontinuous variation of $\left\langle\sigma_{\mathrm{B}}\right\rangle$ is associated to an opposite discontinuous variation of $\left\langle\sigma_{\mathrm{A}}\right\rangle$ (and vice-versa); this is due to the "antiferromagnetic" coupling between the sublattices, and will be illustrated in figure 12 .

2.5 THE PARTICULAR CASE $J_{\mathrm{A}}=J_{\mathrm{B}}=J$. - The point is to distinguish between the symmetrical and non-symmetrical solutions. For this purpose, we introduce the parameters $m, n$ 


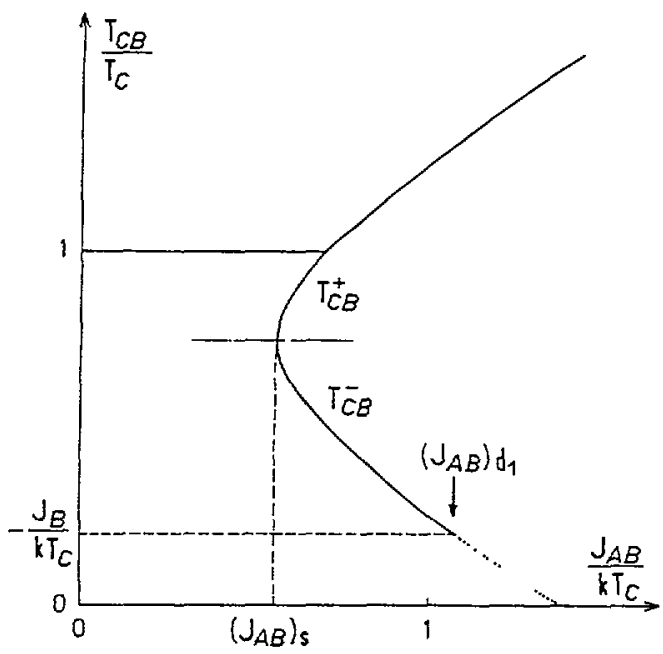

a)

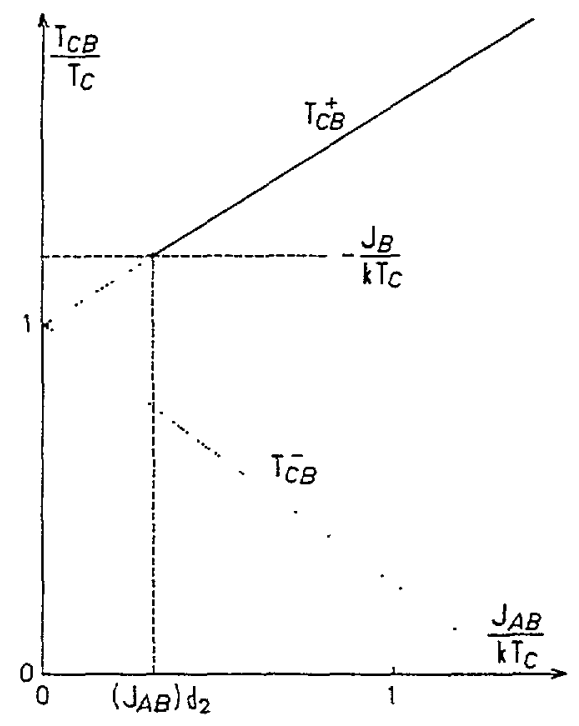

b)

Fig. 6. - Computed values of $T_{\mathrm{CB}}^{ \pm}$, as a function of $J_{\mathrm{AB}}$. The criterion for double-step is $T_{\mathrm{CB}}^{-} \neq T_{\mathrm{CB}}^{+}$. The solid (resp. dotted) line corresponds to a continuous (resp. discontinuous) change of sign. (a) $J_{A}$ $=-0.3 k T_{\mathrm{C}}, J_{\mathrm{B}}=-0.2 k T_{\mathrm{C}}$. (b) $J_{\mathrm{A}}=-1.40 k T_{\mathrm{C}}, J_{\mathrm{B}}=-1.2 \mathrm{k} T_{\mathrm{C}}$.

defined as follows:

$$
\begin{array}{r}
m=\left\langle\sigma_{\mathrm{A}}\right\rangle+\left\langle\sigma_{\mathrm{B}}\right\rangle \\
n=\left\langle\sigma_{\mathrm{A}}\right\rangle-\left\langle\sigma_{\mathrm{B}}\right\rangle
\end{array}
$$

The symmetrical solutions correspond to $n=0$, and non symmetrical solutions to $n \neq 0$. The parameter $\mathbf{n}$ is an order parameter : in terms of magnetism, $n=0$ corresponds to a "saturated paramagnetic" phase, $n \neq 0$ to an "antiferromagnetic" phase. Because of invariance on interchanging $\mathrm{A}$ and $\mathrm{B}$, if a solution $(n \neq 0, m)$ exists, then also $(-n, m)$, with the same free-energy.

Using the new parameters $m, n$, the mean field equations are re-written:

$$
\begin{aligned}
& m=\frac{-2\left(1-r^{2} \exp \left[-2 \beta\left(\Delta+J_{0} \cdot m\right)\right]\right)}{\operatorname{den}} \\
& n=\frac{-4 r \exp \left[-\beta\left(\Delta+J_{0} \cdot m\right)\right]}{\operatorname{den}} \operatorname{sh}(\beta \tilde{J} \cdot n)
\end{aligned}
$$

where $J_{0}=\mathrm{J}+J_{\mathrm{AB}}$

$$
\begin{aligned}
\tilde{J} & =J-J_{\mathrm{AB}} \\
\operatorname{den} & =1+2 r \exp \left[-\beta\left(\Delta+J_{0} \cdot m\right)\right] \operatorname{ch}(\beta \tilde{J} \cdot n)+r^{2} \exp \left[-2 \beta\left(\Delta+J_{0} \cdot m\right)\right]
\end{aligned}
$$




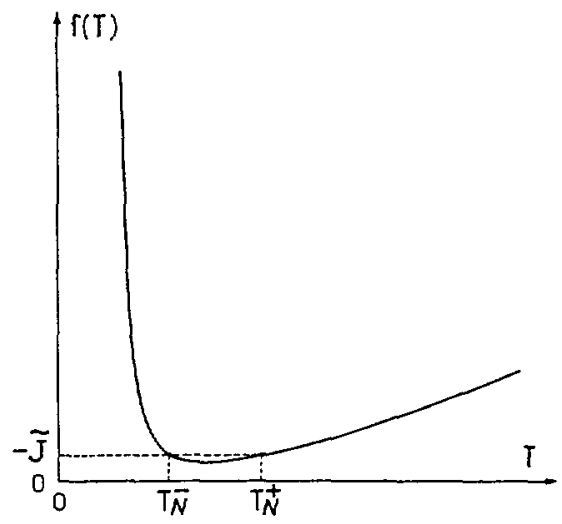

Fig. 7. - Graphic determination of the $2^{\text {nd }}$ order Néel temperatures $T_{\mathrm{N}}^{ \pm}$for $J_{\mathrm{A}}=J_{\mathrm{B}}$, using the plot of equation (14). $T_{\mathrm{N}}^{ \pm}$are the intersects of $f(T)$ with the horizontal line $-\tilde{J}=$ Cst.

A simple inspection of equations $(11,12)$ leads to the following remarks:

(i) the symmetrical solutions always exists.

(ii) if a solution $(n \neq 0, m)$ exists, then $(-n \neq 0, m)$ also does, as mentioned above; they have the same free energy.

We have numerically checked that for $n \neq 0$ there are only two solutions $(\mp n, m)$, and compared the free-energy values of $(0,0)$ and $(\mp n, m)$ solutions (the analytical expression of the free energy is obtained in the Appendix). We concluded that the non-symmetrical solutions (when they exist) are always more stable.

At $0 \mathrm{~K}$ the solution of equations $(11,12)$ is $n=0, m=-2$, and consequently the system starts from a symmetrical situation. A simple differential analysis of the problem allows to determine temperatures $T_{\mathrm{N}}^{ \pm}$corresponding to symmetry breakings associated to continuous variations of $\left\langle\sigma_{\mathrm{A}, \mathrm{B}}\right\rangle$; these are similar to $2^{\text {nd }}$ order Néel temperatures in magnetism:

Let us consider a symmetrical solution $\left(n=0, m_{0}(T) \neq 0\right)$ for a given temperature $\mathrm{T}$. If $T$ is a Néel température, $T_{\mathrm{N}}^{-}$or $T_{\mathrm{N}}^{+}$, then a small temperature variation $\delta T$ (respectively positive or negative) will result, for the non-symmetrical solution, in variations $\delta m, \delta n=n \neq$ 0 . By differenciating equations $(11,12)$, we obtain two linear relations involving $\delta T, \delta m, n^{2}$. We can then show that a solution $n^{2} \neq 0$ requires that:

$$
\left(1+r \exp \left[-\beta\left(\Delta+J_{0} \cdot m_{0}\right)\right]\right)^{2}=-4 r \beta \tilde{J} \exp \left[-\beta\left(\Delta+J_{0} \cdot m_{0}\right)\right]
$$

Equation (13) is conveniently converted into:

$$
f(T)=-\tilde{J}
$$

where:

$$
f(T)=\frac{\left(1+r \exp \left[-\beta\left(\Delta+J_{0} \cdot m_{0}(T)\right)\right]\right)_{2}}{4 r \beta \exp \left[-\beta\left(\Delta+J_{0} \cdot m_{0}(T)\right)\right]}
$$

A typical plot of $f(T)$ is shown in figure 7. This curve has a single minimum value $f_{\min }$; so for - $\tilde{J}>f_{\min }$, the system allows two $2^{\text {nd }}$ order Néel temperatures $T_{\mathrm{N}}^{-}$and $T_{\mathrm{N}}^{+}$. We can argue by continuity, on increasing temperatures from $0 \mathrm{~K}$, that the thermal sequence is: symmetrical, non-symmetrical, symmetrical. So, the low-symmetry , "antiferromagnetic", phase occurs inbetween $T_{\mathrm{N}}^{-}$and $T_{\mathrm{N}}^{+}$, when these temperatures exist. 
In agreement with the present analysis, the computed variations of the specific heat show $\lambda$-shaped discontinuities at $T_{\mathrm{N}}^{-}$and $T_{\mathrm{N}}^{+}$. A typical example is shown in figure 8 , with parameters chosen so as the double-step transition is of continuous-continuous type (discontinuous transitions would prevent calculating the specific heat).

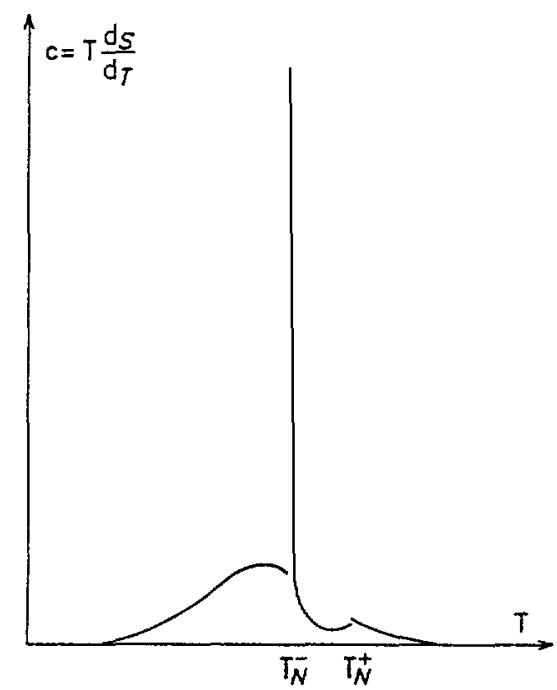

Fig. 8. - Computed specific heat for the particular case $J_{\mathrm{A}}=J_{\mathrm{B}}$. Notice the discontinuities of heat capacity at $T_{N}^{-}$and $T_{N}^{+}$with their different amplitudes; the set of values corresponds to area (22) in the phase diagram of figure $10 \mathrm{~b}$.

In the "antiferromagnetic" phase, the thermal evolutions of the sublattices are different and can be discussed in the way developed for the general case $J_{\mathrm{A}} \neq J_{\mathrm{B}}$. The only thing to do is to label " $B$ " the sublattice the mean value of which changes the more rapidly above $T_{N}^{-}$(this is purely formal since $T_{\mathrm{CA}}^{-}=T_{\mathrm{CB}}^{-}=T_{\mathrm{C}}^{-}, T_{\mathrm{CA}}^{+}=T_{\mathrm{CB}}^{+}=T_{\mathrm{C}}^{+}$). Also, the first-order transition at $T_{\mathrm{C}}$ vanishes.

When equation (13) cannot be satisfied, i.e. when there are no $2^{\text {nd }}$ order Néel transitions, symmetry breakings can yet occur when $\left\langle\sigma_{\mathrm{A}}\right\rangle$ or $\left\langle\sigma_{\mathrm{B}}\right\rangle$ undergo discontinuous variations at $T_{C}^{ \pm}$. The relationships between $T_{\mathrm{N}}^{ \pm}, T_{\mathrm{C}}^{ \pm}$are analyzed in the next section.

2.6 PhaSE DiAgRAM. - The purpose of this section is to analyze simultaneously the one- / two-step, continuous / discontinuous characters of the conversion curve, and also the occurrence of symmetrical or non-symmetrical solutions when $J_{\mathrm{A}}=J_{\mathrm{B}}$. These characters will be described as a function of the interaction parameters $J_{\mathrm{A}}, J_{\mathrm{B}}, J_{\mathrm{AB}}$ in terms of phase diagrams, and the various possibilities for the thermal variations of $\left\langle\sigma_{A}\right\rangle,\left\langle\sigma_{B}\right\rangle,\langle\sigma\rangle$ will be described.

For this purpose, we have done by computer the graphic resolution shown in figure 7 ; the resulting values of $T_{\mathrm{N}}^{ \pm}$have been plotted as a function of $J_{\mathrm{AB}}$ for several values of $J$. Some of the plots are reported in figure 9 .

The comparison of $T_{\mathrm{N}}^{ \pm}$an $T_{\mathrm{C}}^{ \pm}$values, in figure 9 , is really instructive. The Néel values follow a curve of which the upper and lower branches respectively correspond to $T_{\mathrm{N}}^{+}, T_{\mathrm{N}}^{-}$. It is clear that the normal sequence should be: $T_{\mathrm{N}}^{-} \leq T_{\mathrm{C}}^{-} \leq T_{\mathrm{C}}^{+} \leq T_{\mathrm{N}}^{+}$. Consequently, the part of the 


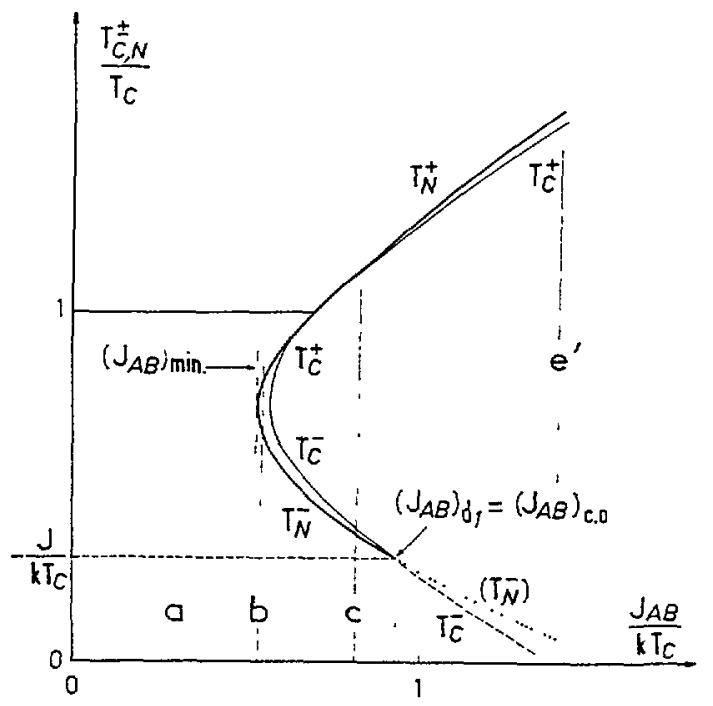

a)

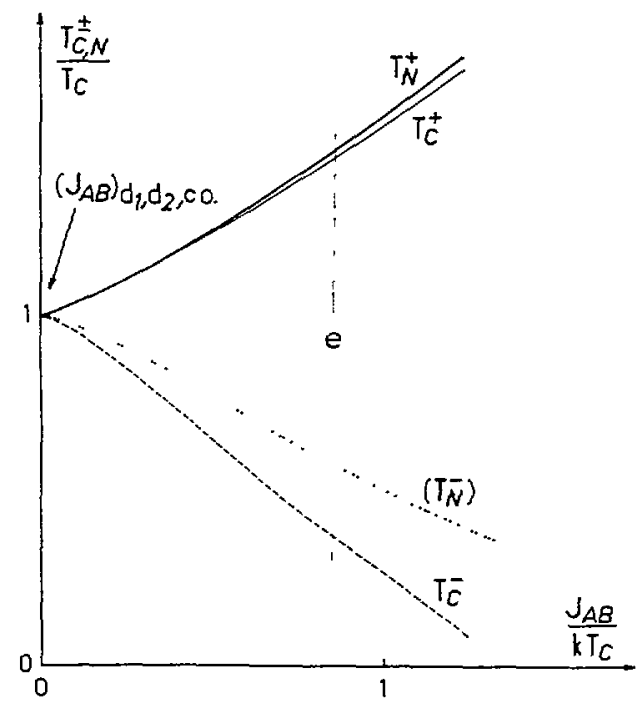

b)

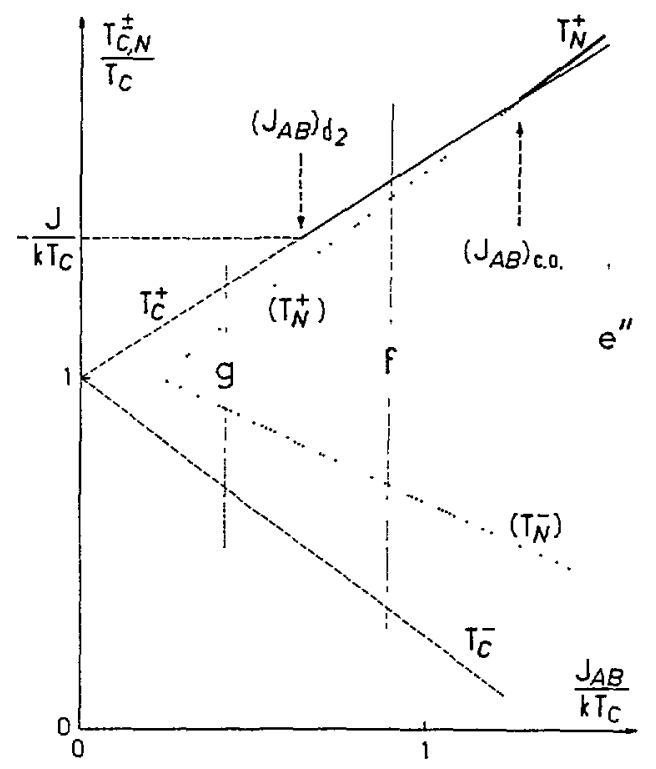

c)

Fig. 9. - Computed values of $T_{\mathrm{C}}^{ \pm}, T_{\mathrm{N}}^{ \pm}$vs. $J_{\mathrm{AB}}$, for the symmetrical case: $J_{\mathrm{A}}=J_{\mathrm{B}}=\mathrm{J}=-0.3$ (a), - 1.0 (b), - 1.4kT $\mathrm{C}$ (c).$T_{\mathrm{C}}^{ \pm}$are represented by thin lines: solid (resp. dashed) when the variations of $\left\langle\sigma_{A, B}\right\rangle$ at zero are continuous (resp. discontinuous). $T_{N}^{ \pm}$are represented by thick solid lines when they effectively correspond to $2^{\text {nd }}$ order Néel transitions $\left(T_{N}^{-}<T_{\mathrm{C}}^{-}, T_{\mathrm{C}}^{+}<T_{\mathrm{N}}^{+}\right)$; by dotted lines when

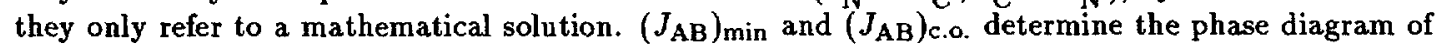
figure $10 \mathrm{~b}$. 
$T_{\mathrm{N}}$ curve which lies inside the $T_{\mathrm{C}}$ curve does not correspond to true Neel temperatures; in this case, the symmetry breakings which are required by $T_{\mathrm{C}}^{-} \neq T_{\mathrm{C}}^{+}$must correspond to $1^{\text {st }}$ order Néel transitions. So, the $1^{\text {st }} / 2^{\text {nd }}$ order of the Néel transitions is monitored by the crossover - which is unique - of the two curves of figure 9 . The corresponding value of $J_{\mathrm{AB}}$ is labelled $\left(J_{\mathrm{AB}}\right)_{\text {c.o. }}$ (c.o. stands for crossover).

It is possible to figure out whether or not these $1^{\text {st }}$ order Néel transitions match the discontinuities at $T_{\mathrm{C}}^{ \pm}$when they exist):

For $|J|<k T_{\mathrm{C}}$ : then $\left(J_{\mathrm{AB}}\right)_{\text {c.o. }} \leq\left(J_{\mathrm{AB}}\right)_{\mathrm{d}_{1}}$ (Fig. 9a corresponds to equality). Two cases are considered:

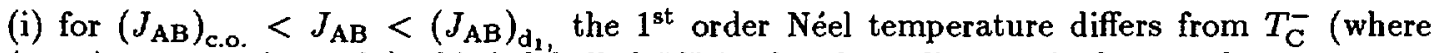
$\left\langle\sigma_{A, B}\right\rangle$ vary continuously); this is labelled " $\mathrm{d}$ " in the phase diagram in figure $10 \mathrm{~b}$;

(ii) for $\left(J_{\mathrm{AB}}\right)_{\mathrm{d}_{1}}<J_{\mathrm{AB}}$, the discontinuity which occurs at $T_{\mathrm{C}}^{-}$can be considered as (and effectively is) a $1^{\text {st }}$ order Néel transition.

For $|J|>k T_{\mathrm{C}}$ : then $\left(J_{\mathrm{AB}}\right)_{\mathrm{d}_{2}}<\left(J_{\mathrm{AB}}\right)_{\text {c.o. }}$ (Fig. 9c). Two cases are considered:

(iii) for $0<J_{\mathrm{AB}}<\left(J_{\mathrm{AB}}\right)_{\mathrm{d}_{2}}$, the discontinuity at $T_{\mathrm{C}}^{+}$can be considered as (and in fact is) a $1^{\text {st }}$ order Néel transition;

(iv) for $\left(J_{\mathrm{AB}}\right)_{\mathrm{d}_{2}}<J_{\mathrm{AB}}<\left(J_{\mathrm{AB}}\right)_{\mathrm{c} . \mathrm{o} .}$, the $1^{\text {st }}$ order Néel temperature differs from $T_{\mathrm{C}}^{+}$(where $\left\langle\sigma_{\mathrm{A}, \mathrm{B}}\right\rangle$ vary continuously); this is " $\mathrm{f}$ " in the phase diagram;

The "valid" part of the $T_{\mathrm{N}}^{ \pm}$curve (which only corresponds to $2^{\text {nd }}$ order transitions!) is drawn as a thick solid line on the plots of figures 9 . It can be noted that the present analysis does not estimate $T_{\mathrm{N}}^{ \pm}$when they are $1^{\text {st }}$ order and differ from $T_{\mathrm{C}}^{ \pm}$.

The determination of the phase diagram in the space $J_{\mathrm{A}}, J_{\mathrm{B}}, J_{\mathrm{AB}}$ would require the construction of a huge number of plots similar to those of figures 9 . In fact, it is sufficient to do it for a given value of $\Delta$ which scales the energies. For convenience, we have presented separately the one- / two -step and continuous / discontinuous characters of the sub-lattice magnetizations when they cross zero (Fig. 10a), and the symmetrical / non-symmetrical and $1^{\text {st }} / 2^{\text {nd }}$ order of the Néel transitions (Fig. $10 \mathrm{~b}, J_{\mathrm{A}}=J_{\mathrm{B}}$ ).

Phase diagrams for $J_{\mathrm{A}} \neq J_{\mathrm{B}}$ (Fig. 10a) have been considered at constant $\frac{J_{\mathrm{B}}}{J_{\mathrm{A}}}$ The line (s) remain unchanged, while lines $\left(\mathrm{d}_{1}, \mathrm{~d}_{2}\right)$ moves upwards when the ratio $\frac{J_{\mathrm{B}}}{J_{\mathrm{A}}}$ is decreased.

Typical thermal variations of $\left\langle\sigma_{\mathrm{A}}\right\rangle,\left\langle\sigma_{\mathrm{B}}\right\rangle,\langle\sigma\rangle$ are shown in Figs.11 for various sets of parameters indicated in the phase diagram of the particular case $J_{\mathrm{A}}=J_{\mathrm{B}}$ (Fig.10b). A systematic inspection of $\langle\sigma\rangle$ shows that the second step of the conversion is always smoother than the first step; this remains true for $J_{\mathrm{A}} \neq J_{\mathrm{B}}$.

With the help of the curves of figure 11, the phase diagram of the symmetrical case (Fig.10b) can be commented as follows: moving from (a) to $(\mathrm{g})$ around the point $J=-k T_{\mathrm{C}}, J_{\mathrm{AB}}=0$, we successively observe

a: a continuous, symmetrical behavior, $\mathbf{a} \rightarrow \mathbf{b}$ : the onset of symmetry breaking $\left(2^{\text {nd }}\right.$ order $)$ below $T_{\mathrm{C}}$, $\mathbf{b} \rightarrow \mathbf{c}$ : the non-symmetrical domain enlarges so that the magnetizations of the sublattices cross the value zero at different temperatures (this is our definition of the "double-step"), $c \rightarrow d$ : the lower Néel temperature becomes $1^{\text {st }}$ order, $\mathbf{d} \rightarrow \mathbf{e}:$ the corresponding discontinuity increases so as to result in a sign change of one sublattice, $\mathbf{e} \rightarrow \mathbf{f :}$ the higher Néel temperature also becomes $1^{\text {st }}$ order,

$\mathbf{f} \rightarrow \mathbf{g}$ : the corresponding discontinuity increases so as to also result in the sign change of the second sublattice.

A plot concerning the general case (with $\frac{J_{\mathrm{B}}}{J_{\mathrm{A}}}=0.8$, corresponding to the label (h) in Fig. 10a) 


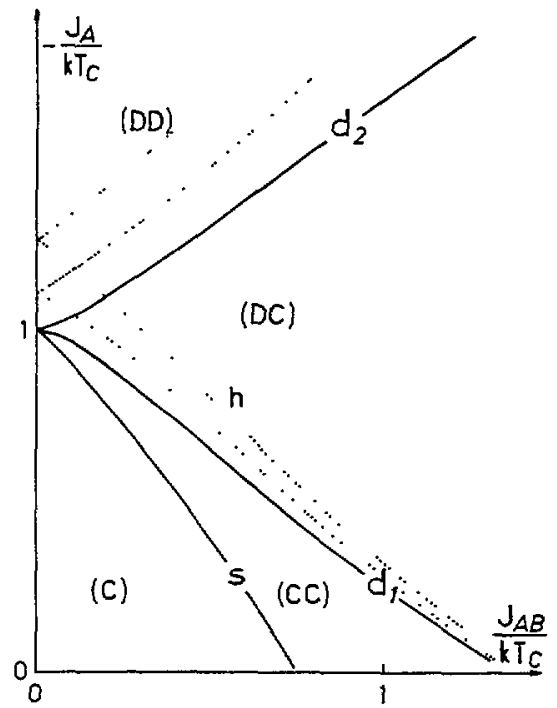

a)

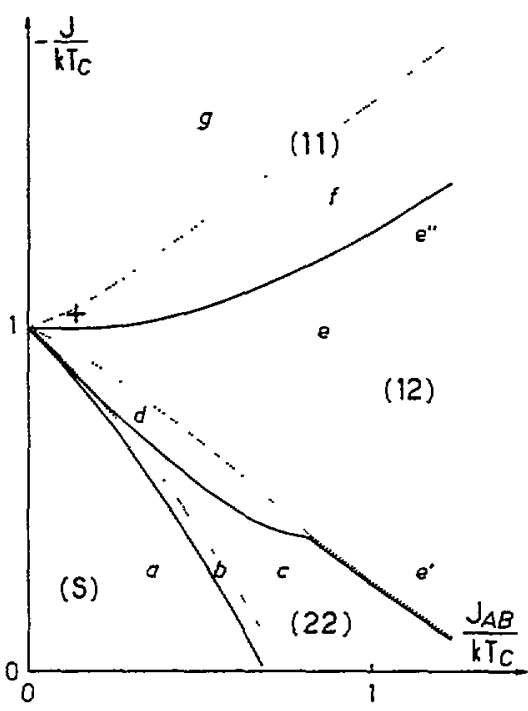

b)

Fig. 10, - a) Phase diagram concerning the continuous / discontinuous and one- / two-step characters of the spin conversion when $\left\langle\sigma_{\mathrm{A}, \mathrm{B}}\right\rangle$ crosses zero. The borderlines correspond to the plots of $\left(\frac{J_{\mathrm{AB}}}{k T_{\mathrm{C}}}\right)_{\mathrm{B}}$, $\left(\frac{J_{\mathrm{AB}}}{k T_{\mathrm{C}}}\right)_{\mathrm{d}_{1}},\left(\frac{J_{\mathrm{AB}}}{k T_{\mathrm{C}}}\right)_{\mathrm{d}_{2}}$ vs $\frac{J_{\mathrm{A}}}{k T_{\mathrm{C}}}$. The symbols $(C),(D)$ stand for the continuous or discontinuous character of the magnetization reversals at $T_{\mathrm{CB}}^{\mp}$. Solid lines correspond to $J_{\mathrm{A}}=J_{\mathrm{B}}$, dotted lines to $\frac{J_{\mathrm{B}}}{J_{\mathrm{A}}}=$ $0.9,0.8$.

b) Phase diagram concerning the occurrence of the non-symmetrical phase and the $1^{\text {st }}$ and $2^{\text {nd }}$ order of the Néel transitions. The borderlines (solid lines) correspond to the plots vs $\frac{J}{k T_{\mathrm{C}}}$ of $\left(J_{\mathrm{AB}}\right)_{\min }$ and of $\left(J_{\mathrm{AB}}\right)_{\text {c.o. }}$ defined in figure 9 . The different areas are labelled in agreement with [7]: (11): both Néel transitions are $1^{\text {st }}$ order, (12) : the lower is $1^{\text {st }}$ order, the higher $2^{\text {nd }}$ order, $(22):$ both are $2^{\text {nd }}$ order. (s) stands for symmetrical at any temperature. The borderlines of the previous phase diagram (Fig.10a) for $J_{\mathrm{A}}=J_{\mathrm{B}}$ have been reproduced as dotted lines. Typical situations, labelled by letters from a to $\mathrm{g}$ are illustrated in figure 11 and described in the forelast paragraph of section 2.6.; two additional situations $\left(e^{\prime}, e^{\prime \prime}\right)$ have been considered in order to complete figures $9 a, c$.

is reproduced in figure $12 \mathrm{in}$ order to illustrate the discontinuities at $T_{\mathrm{C}}$ analyzed in section 2.3.

All these results are summarized in a recapitulary table (Tab. I).

\section{Comparison to previous results.}

In a previous paper, Bari and Sivardière [7] considered the spin conversion of one- and twosublattices (crystallographically equivalent) structures, together with the influence of magnetic interactions. They started from a simple lattice model with elastic and vibronic contributions to the energy. They showed its equivalence to a two-level model characterized by an energy gap depending linearly on the order parameter; this is similar to the present model and leads to equations which are formally similar to ours (i.e. to Wajnflasz and Pick's restricted to two 


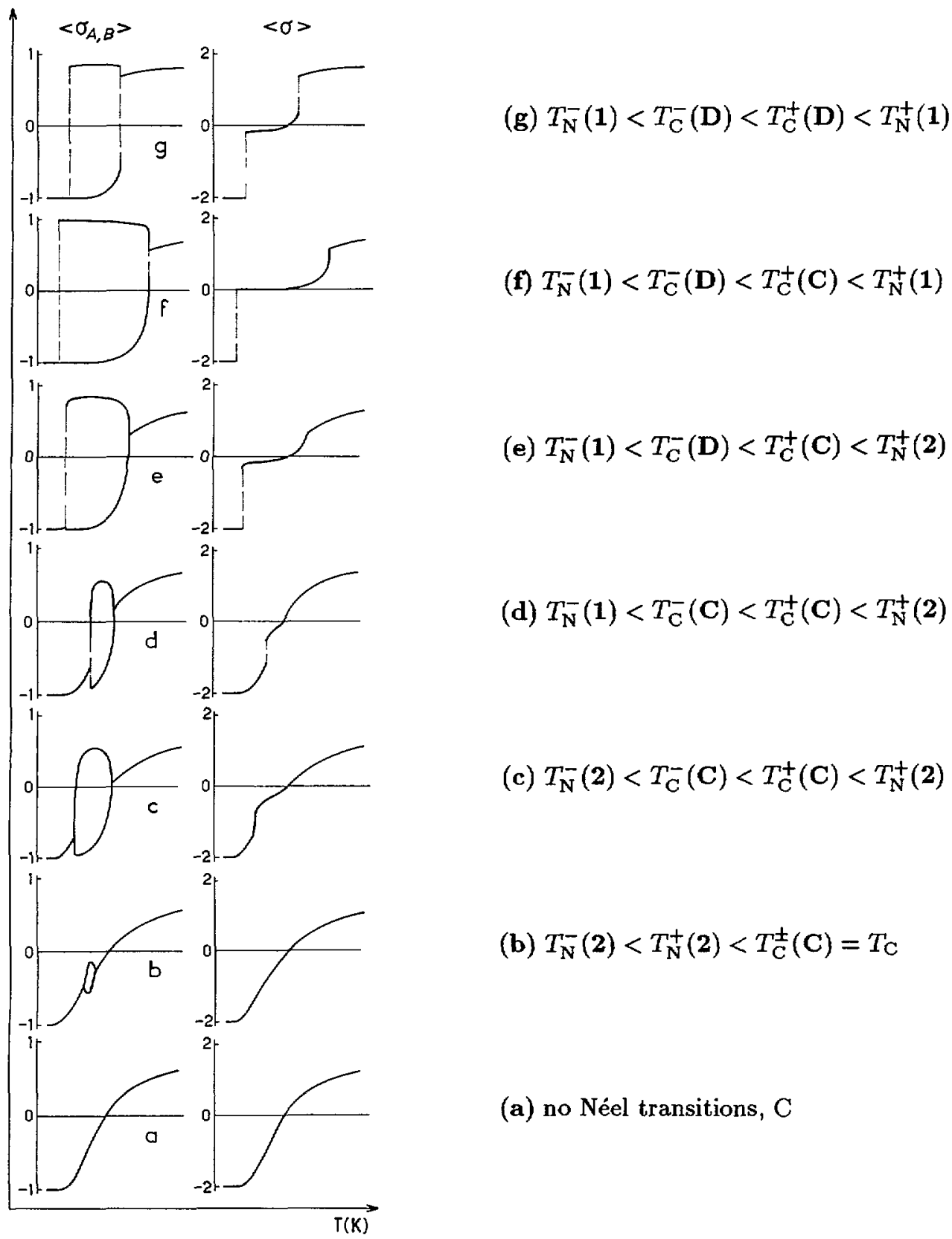

Fig. 11. - Thermal variation of $\left\langle\sigma_{\mathrm{A}}\right\rangle,\left\langle\sigma_{\mathrm{B}}\right\rangle,\langle\sigma\rangle$ for the particular case $J_{\mathrm{A}}=J_{\mathrm{B}}$, in typical cases reported in the phase diagram of figure $10 \mathrm{~b}$ (and schematically reported in figure 9 , excepting situation c). The characters of the Néel transitions, which can be $2^{\text {nd }}$ or $1^{\text {st }}$ order, and of the crossings to zero, continuous or discontinuous, are designed as 2 or $1, C$ or $D$, respectively. 


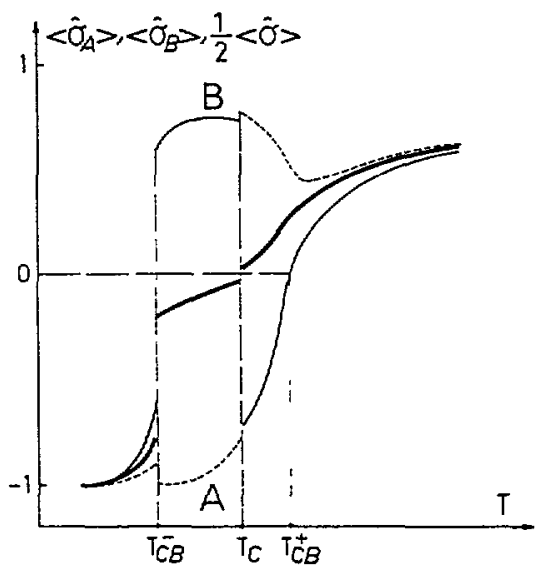

Fig. 12. - Thermal variations of $\left\langle\sigma_{\mathrm{A}}\right\rangle$ (broken line), $\left\langle\sigma_{\mathrm{B}}\right\rangle$ (solid line), $(\sigma)$ (thick solid line), for $J_{\mathrm{A}} \neq$ $J_{B}$, in a situation labelled (h) in figure $10 \mathrm{a}$, showing the discontinuities at $T_{\mathrm{C}}$ and the unequivalence of the sublattices at any temperature. $r=15, J_{\mathrm{A}}, J_{\mathrm{B}}, J_{\mathrm{AB}}=-0.75,-0.60,+0.60 k T_{\mathrm{C}}$ respectively.

levels). They further showed the equivalence to a molecular field treatment with interactions between sites $i, j$ written in terms of occupation numbers $n_{i}, n_{j}$. The eigenvalues of operators $\mathbf{n}$ are 0,1 , and this is the only difference with the Ising-like treatment of W-P, whose $\sigma$ is a spin with eigenvalues \pm 1 . The translation from one to the other treatment is merely obtained by the following formulas:

$$
\begin{aligned}
\left(\sigma_{i}\right)_{\text {here }} & =\left(2 n_{i}-1\right)_{\mathrm{B}-\mathrm{S}} \\
(\Delta)_{\text {here }} & =\left(\Delta-K+\frac{L}{2}\right)_{\mathrm{B}-\mathrm{S}} \\
(J)_{\text {here }} & =\left(-\frac{K}{2}\right)_{\mathrm{B}-\mathrm{S}} \\
\left(J_{\mathrm{AB}}\right)_{\text {here }} & =\left(\frac{L}{4}\right)_{\mathrm{B}-\mathrm{S}}
\end{aligned}
$$

So, the difference between the present treatment and B-S's only lies in the different definitions of the constants. This explains why the transition temperature depends on the interaction parameter in the B-S treatment (at $\Delta_{\mathrm{B}-\mathrm{S}}$ constant), while it does not in the W-P treatment (at $\Delta_{\mathrm{W}-\mathrm{P}}$ constant). There is no way to decide which of W-P or $\mathrm{B}-\mathrm{S}$ treatments is the more physically relevant, since $\Delta, \mathrm{K}$ are independent parameters. In other words, the difference between $\mathrm{B}-\mathrm{S}$ and $\mathrm{W}-\mathrm{P}$ is purely cosmetic.

Consequently, the B-S results on the two-sublattice problem (which only deal with the symmetry breakings) are expected to be identical to ours. Once adapted with the proper translation of constants, their phase diagram is quite similar to ours; they are compared in figure 13. There is however a noticeable difference concerning area (D) of B-S which does not appear in our diagram; it is easily controlled that the threshold value $\left(J_{\mathrm{AB}}\right)_{\mathrm{s}}=0$ for $|J|>$ $k T_{\mathrm{c}}$ (Fig.6b): then $T_{\mathrm{C}}^{-} \neq T_{\mathrm{C}}^{+}$for any $J_{\mathrm{AB}}>0$, which indeed demonstrates the presence of non-symmetrical solutions, and rules out the existence of area (D). 


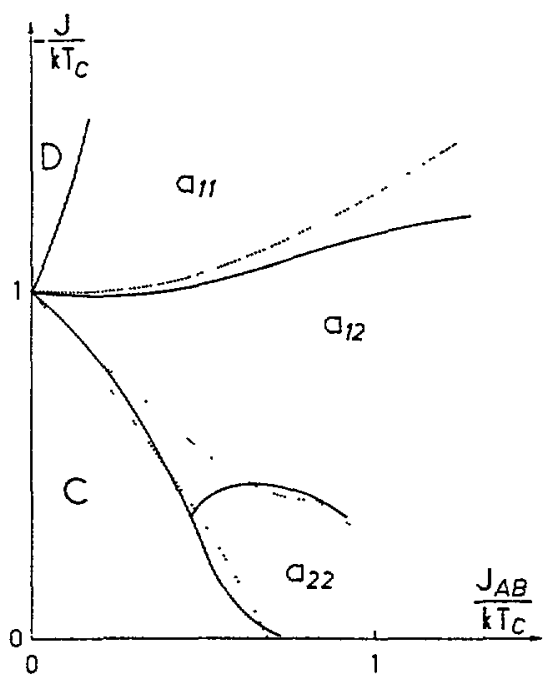

Fig. 13. - Phase diagram adapted from B-S's results (Fig.6 of [7]). Area (D) corresponds to a symmetrical evolution with discontinuous transition (which does not occur in the present analysis).

Such a difference may be due to the numerical approach of B-S, whose accuracy seems to be insufficient to resolve two discontinuities occuring at close temperatures. On the contrary, the present investigation is mostly based on the analytic determination of the characteristic temperatures $T_{\mathrm{C}}^{ \pm}$and $T_{\mathrm{N}}^{ \pm}$; this ensures a good accuracy of the results.

An alternative explanation for the difference might be an hysteresis effect larger than the width of the plateau (this explanation would assume that the solution chosen by B-S was not the thermodynamically most stable at every temperature). Hysteresis effects will be studied separately.

\section{Comparison to experimental data.}

H. Köppen et al. [1] have published a detailed study of the two-step spin conversion in $\left[\mathrm{Fe}[2-\text { pic }]_{3}\right] \mathrm{Cl}_{2}$-EtOH where $(2-$ pic $=2$ - picolylamine $)$. Mössbauer spectroscopy and magnetic susceptibility data are in fair agreement and lead to the plot of $n_{\mathrm{HS}}$ vs. $T$ reproduced in figure 14 .

Using the present model, we have obtained, through a least-square fit procedure the following parameter values: $J_{\mathrm{A}}=J_{\mathrm{B}}=-123 \mathrm{~K}, J_{\mathrm{AB}}=12.1 \mathrm{~K}, \Delta=320 \mathrm{~K}$. We had fixed, following [4], $r=15$ corresponding to the total electronic degeneracies of the ${ }^{1} A_{1}$ and ${ }^{5} \mathrm{~T}_{2}$ states. The most important feature of the plot, i.e. the inflexion around $T_{\mathrm{C}}$ is obtained, and gives evidence of the relevantness of the model. However, the high temperature values are not well reproduced (dotted line in Fig.14) and require a significant change in the value of $r:$ indeed, the high temperature limit of $\langle\sigma\rangle$ is $2\left(\frac{r-1}{r+1}\right)$, and consequently, the high temperature limit of $n_{\mathrm{HS}}=$ $\frac{1}{4}\langle\sigma\rangle+0.5$ is $\frac{r}{r+1}$; the value $r=400$ greatly improves the fit (solid line in Fig.14). Then $\Delta$ $=705 \mathrm{~K}, J_{\mathrm{A}}=J_{\mathrm{B}}=-123 \mathrm{~K}, J_{\mathrm{AB}}=16.7 \mathrm{~K}$. This set of values corresponds to a situation of (DC,11) type, reported as a cross in Fig.10b). Then the computed characteristic temperatures 


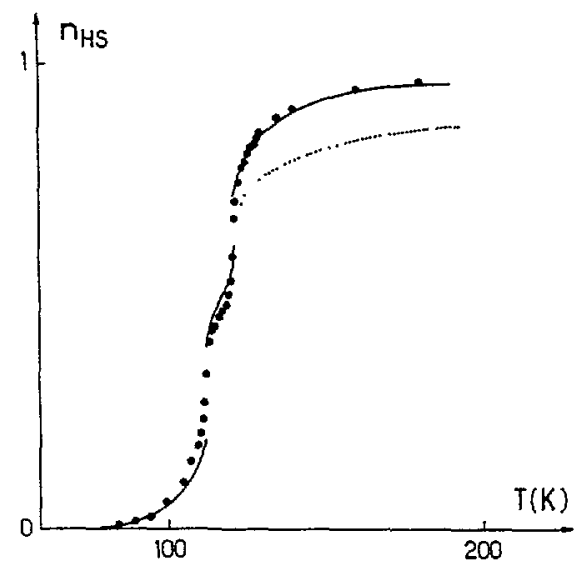

Fig. 14. - Experimental values (crosses) of the high-spin fraction $n_{\mathrm{HS}}(T)$, for [ $\mathrm{Fe}^{\mathrm{II}}[2-\mathrm{pic}] 3 \mathrm{Cl}_{2}$ -EtOH, from [1], and least-square fits with $r=15$ (dotted line), 400 (solid line).

are: $T_{\mathrm{C}}^{-}=T_{\mathrm{N}}^{-}=113.8 \mathrm{~K}, T_{\mathrm{C}}=117.7 \mathrm{~K}, T_{\mathrm{C}}^{+}=121.2 \mathrm{~K} \lesssim T_{\mathrm{N}}^{+}$(the $1^{\text {st }}$ order Néel temperatures are not analytically determined since they are not solutions of Eq. (13)).

( $A$ reason for increasing $r$ might lie in the vibrational entropies which strongly differ in the $L S$ and HS states. This will be discussed in a separate report).

The specific heat corresponding to the fitted set of values should exhibit a divergence at $T_{\mathrm{N}}^{-}$ $=T_{\mathrm{C}}^{-}$, a finite peak at $T_{\mathrm{C}}^{+}$, and a smaller divergence at $T_{\mathrm{N}}^{+}$very close to $T_{C}^{+}$. Practically, a first, narrow and high peak and a second, broad and low, one are then expected. The experimental data [8] show the reverse. This will be discussed below.

A second example is that of $\mathrm{Fe}^{\mathrm{II}}\left(5 \mathrm{NO}_{2}-\mathrm{sal}-\mathrm{N}(1,4,7,10)\right)$ [2a] for which we have used the Mössbauer data of $n_{\mathrm{HS}}(T)$ (Fig.15). The best fit is obtained with $r=400, J_{\mathrm{A}}=J_{\mathrm{B}}=-280$ $\mathrm{K}, J_{\mathrm{AB}}=29 \mathrm{~K}, \Delta=920 \mathrm{~K}$. The conversion proceeds through 2 discontinuous steps and leads to a thermal hysteresis which has been observed on the magnetic data. In the phase diagram the corresponding point lies far away near the vertical axis, in the (11), (DD) zone, such that $T_{\mathrm{N}}^{-}=T_{\mathrm{C}}^{-}, T_{\mathrm{N}}^{+}=T_{\mathrm{C}}^{+}$.

It can be remarked, for both experimental data analyzed here, that the second step is steeper than the first one; this results in sizable misfits in figures 14,15 , and suggests that the assumption $\Delta_{\mathrm{A}}=\Delta_{\mathrm{B}}$ is not so well fulfilled. A more general analysis of the data, involving $\Delta_{\mathrm{A}} \neq \Delta_{\mathrm{B}}$, should also account for the thermal hysteresis, and will be undertaken in a further step.

A similar analysis in the case of the previous complex might also explain the discrepancy between experimental and computed specific heat, since the shapes of the $C_{p}$ anomalies are clearly related to the steepnesses of the conversion curve at the characteristic temperatures.

\section{Conclusion.}

We have described the two-step spin-conversion with a very simple model based on four parameters only: $T_{C}$ which is a characteristic temperature of the molecular system, and three "exchange" parameters $J_{\mathrm{A}}, J_{\mathrm{B}}, J_{\mathrm{AB}}$. The intra-sublattice parameters $J_{\mathrm{A}}, J_{\mathrm{B}}$ are negative ("ferromagnetic" interactions) and the inter-sublattice parameter $J_{\mathrm{AB}}$ is positive ("antiferro- 


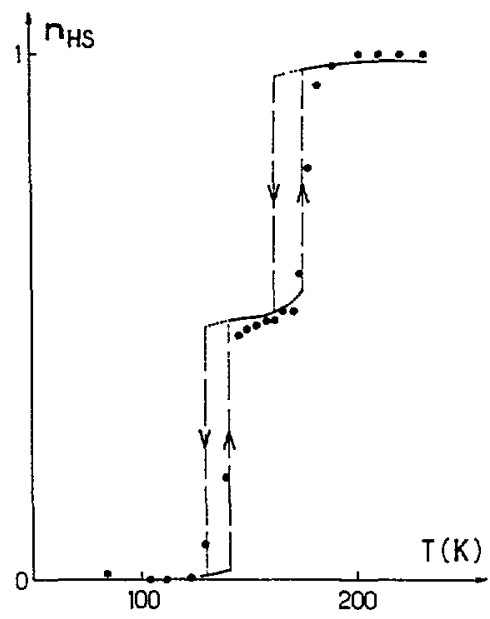

Fig. 15. - Experimental values of $\mathrm{Fe}^{\mathrm{II}}\left[5 \mathrm{NO}_{2}-\mathrm{sal}-(1,4,7,10)\right]$, from [2a], and least square fit with $r=400$

magnetic" interaction). The two-step evolution results from the coupled thermal variations of the sublattices near $T_{C}$; it is predicted that, in any case, the first step is steeper than the second one, and this clearly is a consequence of the assumption $\Delta_{\mathrm{A}}=\Delta_{\mathrm{B}}$.

The model also predicts in the general case $\left(J_{\mathrm{A}} \neq J_{\mathrm{B}}\right)$ a reversal of the "magnetizations" of the sublattices at $T_{C}$, with the thermodynamical properties of a first-order transition. In the particular case of equivalent sublattices $\left(J_{\mathrm{A}}=J_{\mathrm{B}}\right)$ this first-order transition at $T_{\mathrm{C}}$ vanishes, but an "antiferromagnetic" phase between two Néel temperatures $T_{\mathrm{N}}^{-}$and $T_{\mathrm{N}}^{+}$can occur; the Néel transitions can be of $2^{\text {nd }}$ or $1^{\text {st }}$ order.

These features have been investigated simultaneously, by using analytic approaches which ensure the reliability of the final phase diagram.

Finally, we have well reproduced the experimentally observed two-step spin conversions; the predictions concerning the discontinuities of specific heat at $T_{N}^{\mp}$ and the reversals at $T_{\mathrm{C}}$ remain to be observed. The present model could be applied more widely to two-level two-sublattice systems, for which other examples should be found among molecular solids.

We are indebted to the referees of Journal de Physique, whose constructive remarks have been appreciated.

\section{Appendix}

Calculation of the free-energy, entropy and heat capacity in the mean-field approximation.

\section{General EXPRESSION OF $F$.}

It is well known [6] that, $F$, the free-energy of the system of $N$ molecules in the mean-field approximation is given by

$$
F=-\frac{N}{2} k T\left[\operatorname{Ln}\left(Z_{0 \mathrm{~A}}\right)+\operatorname{Ln}\left(Z_{0 \mathrm{~B}}\right)\right]-\frac{N}{4}\left(J_{\mathrm{A}}\left\langle\sigma_{\mathrm{A}}\right\rangle^{2}+J_{\mathrm{B}}\left\langle\sigma_{\mathrm{B}}\right\rangle^{2}+2 J_{\mathrm{AB}}\left\langle\sigma_{\mathrm{A}}\right\rangle \cdot\left\langle\sigma_{\mathrm{B}}\right\rangle\right)
$$


with

$$
\begin{aligned}
& Z_{0 \mathrm{~A}}=g_{\mathrm{a}} \exp \beta\left[\frac{\Delta}{2}+J_{\mathrm{A}}\left\langle\sigma_{\mathrm{A}}\right\rangle+J_{\mathrm{AB}}\left\langle\sigma_{\mathrm{B}}\right\rangle\right]+g_{\mathrm{b}} \exp -\beta\left[\frac{\Delta}{2}+J_{\mathrm{A}}\left\langle\sigma_{\mathrm{A}}\right\rangle+J_{\mathrm{AB}}\left\langle\sigma_{\mathrm{B}}\right\rangle\right] \\
& Z_{0 \mathrm{~B}}=g_{\mathrm{a}} \exp \beta\left[\frac{\Delta}{2}+J_{\mathrm{B}}\left\langle\sigma_{\mathrm{B}}\right\rangle+J_{\mathrm{AB}}\left\langle\sigma_{\mathrm{A}}\right\rangle\right]+g_{\mathrm{b}} \exp -\beta\left[\frac{\Delta}{2}+J_{\mathrm{B}}\left\langle\sigma_{\mathrm{B}}\right\rangle+J_{\mathrm{AB}}\left\langle\sigma_{\mathrm{A}}\right\rangle\right]
\end{aligned}
$$

and $\beta=(k T)^{-1}$

\section{VALUE AT $T_{\mathrm{C}}$.}

The characteristic value $T_{C}$ is defined by

$$
\begin{aligned}
r \exp \left(-\beta_{\mathrm{C}} \Delta\right)=1 \quad \text { with } \quad r=\frac{g_{\mathrm{b}}}{g_{\mathrm{a}}} \\
F\left(T_{\mathrm{C}}\right)=-N k T_{\mathrm{C}} \operatorname{Ln}\left(g_{\mathrm{a}}\right)-\frac{N}{2} \Delta \\
-\frac{N}{2} k T_{\mathrm{C}}\left\{2 \operatorname{Ln}(2)+\operatorname{Ln}\left(\operatorname{ch}\left[\beta_{\mathrm{C}}\left(J_{\mathrm{A}}\left\langle\sigma_{\mathrm{A}}\right\rangle+J_{\mathrm{AB}}\left\langle\sigma_{\mathrm{B}}\right\rangle\right)\right]\right)\right\} \\
-\frac{N}{2} k T_{\mathrm{C}} \operatorname{Ln}\left(\operatorname{ch}\left[\beta_{\mathrm{C}}\left[J_{\mathrm{B}}\left\langle\sigma_{\mathrm{B}}\right\rangle+J_{\mathrm{AB}}\left\langle\sigma_{\mathrm{A}}\right)\right]\right]\right) \\
-\frac{N}{2}\left\{J_{\mathrm{A}}\left\langle\sigma_{\mathrm{A}}\right\rangle^{2}+J_{\mathrm{B}}\left\langle\sigma_{\mathrm{B}}\right\rangle^{2}+2 J_{\mathrm{AB}}\left\langle\sigma_{\mathrm{A}}\right\rangle \cdot\left\langle\sigma_{\mathrm{B}}\right\rangle\right\}
\end{aligned}
$$

This expression clearly shows that $F\left(T_{\mathrm{C}}\right)$ does not change when $\left(\left\langle\sigma_{\mathrm{A}}\right\rangle,\left\langle\sigma_{\mathrm{B}}\right\rangle\right)$ is replaced by $\left(-\left\langle\sigma_{A}\right\rangle,-\left\langle\sigma_{B}\right\rangle\right)$.

3. PARTiCUlar CASE $J_{A}=J_{B}=J$.

We re-write $F$ by using the following relations

$$
\begin{array}{cc}
J_{0}=J+J_{\mathrm{AB}} & \tilde{J}=J-J_{\mathrm{AB}} \\
\left\langle\sigma_{\mathrm{A}}\right\rangle=\frac{m+n}{2} & \left\langle\sigma_{\mathrm{B}}\right\rangle=\frac{m-n}{2}
\end{array}
$$

we obtain

$$
\begin{aligned}
& Z_{0 \mathrm{~A}}=g_{\mathrm{a}} \exp \left(\beta \frac{\Delta}{2}\right)\left[\exp \frac{\beta}{2}\left(J_{0} \cdot m+\tilde{J} \cdot n\right)+r \exp (-\beta \cdot \Delta) \exp \frac{-\beta}{2}\left(J_{0} \cdot m+\tilde{J} \cdot n\right)\right] \\
& Z_{\mathrm{OB}}=g_{\mathrm{a}} \exp \left(\beta \frac{\Delta}{2}\right)\left[\exp \frac{\beta}{2}\left(J_{0} \cdot m-\tilde{J} \cdot n\right)+r \exp (-\beta \cdot \Delta) \exp \frac{-\beta}{2}\left(J_{0} \cdot m-\tilde{J} \cdot n\right)\right]
\end{aligned}
$$

and

$$
\begin{aligned}
F & =-N k T \operatorname{Ln}\left(g_{\mathrm{a}}\right)-\frac{N}{2} \Delta \\
& -\frac{N}{2} k T\left\{\operatorname{Ln}\left[\exp \frac{\beta}{2}\left(J_{0} \cdot m+\tilde{J} \cdot n\right)+r \exp (-\beta \cdot \Delta) \exp \frac{-\beta}{2}\left(J_{0} \cdot m+\tilde{J} \cdot n\right)\right]\right. \\
& \left.+\operatorname{Ln}\left[\exp \frac{\beta}{2}\left(J_{0} \cdot m-\tilde{J} \cdot n\right)+r \exp (-\beta \cdot \Delta) \exp -\frac{\beta}{2}\left(J_{0} \cdot m-\tilde{J} \cdot n\right)\right]\right\} \\
& -\frac{N}{4}\left(J_{0} \cdot \frac{m^{2}}{2}+\tilde{J} \cdot \frac{n^{2}}{2}\right)
\end{aligned}
$$




\section{ENTROPY.}

$S$ has been obtained analytically in the particular case $J_{\mathrm{A}}=J_{\mathrm{B}}$ :

$$
S=N \operatorname{Ln}\left(\frac{D}{2}\right)+\beta N\left(\Delta+J_{0} \cdot m\right)\left(1-\frac{1+\exp \left(-2 \beta\left(\Delta+J_{0} \cdot m\right)\right)}{D}\right)+\frac{\beta N \tilde{J} n^{2}}{4}
$$

with:

$$
D=1+2 r \exp \left(-\beta\left(\Delta+J_{0} \cdot m\right)\right) \operatorname{ch}\left(\beta \tilde{J} n^{2}\right)+r^{2} \exp \left(-2 \beta\left(\Delta+J_{0} \cdot m\right)\right)
$$

\section{HEAT CAPACITY.}

$C$ is numerically derived from the entropy, according to $C=T \frac{\mathrm{d} S}{\mathrm{~d} T}$

\section{References}

[1] a) Köppen H., Müller E.W., Köhler C.P., Spiering H., Meissner E. and Gütlich P., Chem. Phys. Lett. 91 (1982) 348;

b) Gütlich P. and Hauser A., Coord. Chem. Rev. 97 (1990) 1.

[2] a) Petrouleas V., Tuchagues J.P., Chem. Phys. Lett. 137 (1987) 21;

b) Rakotonandrasana A., Boinnard A., Savariaud J.M., Tuchagues J.P., Petrouleas V., Cartier C., Verdaguer M., Inorg. Chim. Acta, 180 (1991) 19.

[3] Real J., Bolvin H., Bousseksou A., Dworkin A., Kahn O., Varret F. and Zarembowitch J. (to appear in J. Am. Chem. Soc.)

[4] a) Wajnflasz J. and Pick R., J. Phys.Colloq. France 32 (1971) C1-91;

b) Wajnflasz J., Phys. Status Solidi 40 (1970) 537.

[5] a) Goodwin H.A, Coord. Chem. Rev. 18 (1976) 293;

b) Gütlich P., Struct. Bonding 44 (1981) 83;

c) Gütlich P., Adv. Chem. Ser. 194 (1981) 405;

d) König E., Ber. Bunsenges. Phys. Chem. 76 (1972) 975;

e) König E., Ritter G., Mössbauer Effect Methodology, 9 (1974) 1;

f) Gütlich P., In Mössbauer Spectroscopy Applied to Inorganic Chemistry, G.J. Long Ed. (Plenum Press, New York, 1984);

g) Rao ,C.N.R., Int. Rev. Phys. Chem. 4 (1985) 19;

h) König E., Ritter G., Kulshrestha S.K., Chem. Rev. 85 (1985) 219;

i) König E., Prog. Inorg. Chem., vol.35, J. Wiley Ed. (Interscience, 1987).

[6] Boccara N., Symétries Brisées, Théorie des transitions avec paramètre d'ordre (Hermann, Paris, 1976).

[7] Bari R.A., Sivardière J., Phys. Rev. B5 (1972) 4466.

[8] Jakobi R., Spiering H., Gütlich P., J. Phys. Chem. Sol. 53 (1992) 267. 